Hypersensitivity of rods detached from frog retinas. Katsu AZUMA, Dept. of Biol., Osaka Med. Coll., Takatsuki, Osaka 569

Effects of $\left[\mathrm{Ca}^{2+}\right]$ on the light sensitivity of rods mechanically detached from the frog Rana catesbeiana retinas were investigated by sucking the inner and outer segments into tightly fitting pipettes.

Light flashes ( $500 \mathrm{~nm}, 1$ sec duration) evoked transient outward changes of membrane currents (photoresponses). Peak amplitudes of photoresponses to flashes of increasing intensity increased to reach a maximal level. Further increases in the light intensity prolonged the duration of the maximal level. The peak amplitude of maximal response in $2+$ Ringer solution varied between 6 and $12 \mathrm{pA}$.

Reducing $\left[\mathrm{Ca}^{2+}\right.$ ] from $0.9 \mathrm{mM}$ (normal Ca) to $90 \mu \mathrm{M}$ (low $\mathrm{Ca}$ ) influenced the amplitude and time Course of photoresponses evoked by the same intensity flash,i.e., low $\mathrm{Ca}$ induced an increase in the amplitude and a shortening in the recovery phase.

At normal $\mathrm{Ca}$, the onset of moderate adaptation light produced a steady outward change of membrane currents which tended to recover to the initial dark level at the offset of the light. On the other hand, at low Ca, the offset of the light induced a large inward change of membrane current which transiently overshot the initial dark level. In the overshoot state, which was caused by a transient increase of membrane current, flash lights evoked photoresponses which were about 1.2-1.4 times larger than those in the initial dark (called hypersensitivity). Thus hypersensitivity was observed in isolated rods. These results were consistent with the previous measurements of $\left[\mathrm{K}^{+}\right]_{0}$ in the interstitial space of frog retina (1). Several workers have found that in low $\mathrm{Ca}$, light adaptation induces, an increase in $\mathrm{Na}$-gradient across rod cell membrane and besides a decrease in $\left[\mathrm{Ca}^{2+}\right]_{i}$ which causes an increase of gNa(negative feed back). It is discussed that these effects are responsible for the hypersensitivity of rod photoreceptors.

1) K. Azuma, Zoological Science (1988) 5, 33-42

Activation of transducin by phosphorylated metarhodopsin II in the presence of $48 \mathrm{kDa}$ protein. Yoshitaka FUKADA, Tôru Yoshizawa, Hiroshi OHGURO^, Tetsuya Saito* and Toyoaki Akino ${ }^{*}$ Dept. Biophys., Fac. Sci., Kyoto Univ. and *'Dept. Biochem., Sapporo Medical College

In rod outer segment (ROS), a photobleaching intermediate of rhodopsin (Rh), metarhodopsin II (Meta II) couples with transducin ( $T$; GTP-binding protein) for activation of CGMP-phosphodiesterase (PDE), revealing a remarkable amplification (ON-process). The activated phosphodiesterase is subsequently deactivated by phosphorylation of Meta II in the presence of $48 \mathrm{kDa}$ protein (OFF-process). With regard to the OFF-process, two hypotheses are proposed on the basis of measurements of the PDE activity as follows: (i) $48 \mathrm{kDa}$ protein has a higher affinity for phosphorylated Meta II than $T$ does, and the preferential binding of $48 \mathrm{kDa}$ protein to phosphorylated Meta II causes an inhibition on the interaction between the Heta II and T. (ii) $48 \mathrm{kDa}$ protein is activated by phosphorylated Meta II, and the activated $48 \mathrm{kDa}$ protein directly interacts with the active PDE to quench the activity. In order to examine which hypotheses is correct, a stimulation of GTP binding to $T$ by phosphorylated Meta II in the absence or presence of $48 \mathrm{kDa}$ protein were investigated. If the hypothesis (i) is correct, the GTP binding to $T$ must be inhibited by $48 \mathrm{kDa}$ protein, which should be a direct evidence of the mechanism. For the purpose of simplification, phosphorylated bovine Rh was isolated and then incorporated into liposomes with purified $\mathrm{T}$ and $48 \mathrm{kDa}$ protein. After confirming that the purified $48 \mathrm{kDa}$ protein could shorten the lifetime of activated PDE in ROS membranes in the presence of ATP, the GppNHp (non-hydrolyzable analogue of GTP) binding activity of $T$ was measured with or without $48 \mathrm{kDa}$ protein in the reconstituted system. Then, the binding during a constant period was not inhibited by $48 \mathrm{kDa}$ protein which was added in several molar excess to phosphorylated Meta II. Furthermore, initial velocity of the binding was also unaffected by the addition of $48 \mathrm{kDa}$ protein. These observations demonstrate that $48 \mathrm{kDa}$ protein does not inhibit the interaction between phosphorylated Meta II and T. Accordingly, the hypothesis (i) is unlikely to be the mechanism of the OFF-process. 
Ion selective effects on the spindle potential in frog muscle spindle. Fumio ITO, Masahiro SOKABE, Noriaki FUJITSUKA, Keiji NARUSE and Takashi KATO Dept. Physiol., Nagoya Univ. Sch. Med.

The stretch responses of the sensory terminal of muscle spindles isolated from semitendinosus muscles of bull-frog were recorded after partial digestion of the spindle capsule with collagenase. The amplitudes of peak and static components of the spindle potential during a ramp-and-hold stretch were measured in isotonic solutions containing different kinds of mono-, di- and tri-valent cations, and compared with those in an isotonic $\mathrm{NaCl}$ solution containing tetrodotoxin. Time-dependent decreases in the spindle potential without significant differences between the peak and static components were found. The ion permeability sequence found from measurement of the amplitude of the spindle potential was Li $>$ TEA > Tris > TMA > choline > glucosamine > L-arginine. The amplitudes of the spindle potential were increased $2-3$ times in isotonic $\mathrm{NaCl}$ solutions containing $10 \mathrm{~mm}$ $\mathrm{Ba}^{2+}, \mathrm{Ca}^{2+}$ or $\mathrm{Sr}^{2+}$ which were caused by an increase in the trans-gap impedance, but decreased in solutions containing $\mathrm{Mn}^{2+}, \mathrm{Cd}^{2+}, \mathrm{La}^{3+}, \mathrm{Gd}^{3+}, \mathrm{Nd}^{3+}$, verapamil, nefedipine and $\mathrm{N}$-methyl $\mathrm{D}$ - glucamine. These results suggest that the current inducing the spindle potential is due to a large cation channel.

Ocellar outputs to the thoracic nervous system in the American cockroach. Takahiro OHYAMA and Yoshihiro TOH. Dept. Biol., Fac. Sci., Kyushu Univ.

The cockroach, Periplaneta americana, possesses two ocelli in addition to the compound eyes. In order to elucidate ocellar function, we investigated ocellar outputs to the thoracic nervous system which controls the wing and leg motor system. In the present study, two descending ocellar interneurons were identified: they were referred to here as a descending ipsilateral ocellar interneuron (DIO neuron) and a descending contralateral ocellar interneuron (DCO neuron). They have a cell body in the protocerebrum and extend dendritic processes into the ocellar nerve ipsilateral to the cell body. The DIo neuron sends an axon ipsilaterally toward the metathoracic ganglion. The DCO neuron sends an axon across the mid-line of the protocerebrum toward the second abdominal ganglion. Both DIO and DCO axons extend many branches in each ganglion. The DIO and DCO neurons responded with a few off-spikes to illumination of the ocellus ipsilateral to their cell bodies. Simultaneous recording of the ocellar second order neuron and the DCO neuron showed that spikes of the second order neuron caused spikes of the DCO neuron. Both DIO and DCO neurons responded to cercal stimulation with a train of spikes, which were suppressed by ocellar illumination. They also responded with spike discharges to other mechanical stimuli such as brushing the antenna or abdomen by a fine needle. Identification of thoracic interneurons receiving inputs from DIO and DCO neurons were carried out, and one type of interneuron was identified, which was referred to here as a thoracic interganglionic ocellar interneuron (TIO neuron). The TIO neuron has a cell body in the metathoracic ganglion and sends an axon across the mid-line to the mesothoracic ganglion and to the abdominal ganglion. EPSPs were caused in the TIO neuron by spikes of the DCO neuron. The TIO neuron received more synaptic inputs from unknown neurons. Moreover, it responded to cercal stimulation with oscillation of membrane potentials superimposed on depolarization: such oscillation is common in neurons of the insect motor system. The present data suggest descending information would be integrated together with other in the TIO neuron, which would concern delicate modulation of the motor system. 
Signal transmission between second- and third-order neurons of a cockroach ocellus. Makoto MIzUNAMI. Dept. Biol., Kyushu Univ., Fukuoka 812.

Synaptic transmission between second- and third-order neurons of the ocellus of the cockroach, Periplaneta americana, was studied by simultaneous intracellular recordings. Stimulus was a current pulse applied to a second-order neuron or a sinusoidally-modulated light around a mean illuminance. Active components of the response of pre- and postsynaptic neurons were eliminated by applying TTX to analyze the graded responses. Synaptic transmission between second- and third-order neurons is sine non-inverting (excitatory). The normal membrane potentials of second-order neurons are just above the transmission threshold, and sustained transmission is maintained under normal membrane potentials. The synapse has sigmoidal transfer characteristics with an exponentially-growing part near the transmission threshold, and has low-pass filtering characteristics with a cut-off frequency of about $15 \mathrm{~Hz}$. Analysis using a sinusoidally-modulated light or a step incre/decremental light showed that the response of second- and third-order neurons to a modulation input is fundamentally different. First, the response of second-order neurons linearly relates to the depth of modulation, whereas that of third-order neurons is nonlinear. Second, the response of third-order neurons has sharper band-pass filtering characteristics than that of second-order neurons. Third, time-course of the response of third-order neurons is more transient than that of secondorder neurons. These differences are well explained by an exponential transfer characteristics of the synapse between these neurons: signal transmission occurs using exponentially-growing part of the over-all sigmoidal transfer function. I conclude that the signal transmission at the synapse between second- and thirdorder neurons is fundamentally different from that at the synapse between photoreceptors and second-order visual neurons, where linear signal transmission occurs using a linear part of the sigmoidal transfer function.

Firing pattern of the motoneuron mediating the stinging response in the honeybee. Hiroto OGAWA and Tsuneo YAMAGUCHI Dept. Biol., Fac. Sci.., Okayama Univ.

The sting of the honeybee comprizes a stylet and paired lancets. In the stinging response, a set of muscles (a protractor, M198 and a retractor, M199) on each side slide the lancets on the stylet. Recording of electrical activities from these muscles showed that during the stinging response, the protractor and retractor muscles on each side contract alternately, and the protractor muscles and retractor muscles both sides contract out of phase respectively. There was a linear relation between the duration of electrical discharge (DED) and the cycle length of successive electrical discharges (CLD) in all mucsles: both DED and CLD lengthened linearly as the sting inserted deeper into a soft object and the tension in the sting was increased. When the sting was cut at its basal arms on either side to permit the muscular movement without receiving the proprioceptive inputs from the cut side, both DED and CLD were longer in the muscles on the cut side than in those on the opposite side, though DED and CLD on both sides remained correlative. When the sensory nerves running from the sting were cut on both sides, the electrical activities with the shortest DED and CLD were observed irrespective of the strength of tension in the sting. It was suggested, therefore, that two pattern generators, each of which controls the rhythmic movement of the muscles on its own side for the stinging response, exists separately in each half of terminal abdomeninal ganglion, and they were usually coupled with each other to operate. Presumably, both of the rhythmicity produced in each pattern generator and the degree of coupling between the pattern generators are under the strong influence of proprioceptive inputs from the sting. 


\section{S-S loops in the giant hemoglobin of Tylorrhynchus heterochaetus}

Tomohiko SUZUKI*, Oscar H. KAPP** and Toshio GOTOH***

*Dept. Biol., Fac., Sci., Kochi Univ., **Enrico Fermi Inst., Univ. Chicago, $\star \star \star$ Dep. Biol. College of General Education, Univ. Tokushima.

The extracellular hemoglobin of the polychaete Tylorrhynchus heterochaetus has a molecular weight of $3,370 \mathrm{kDa}$, and consists of two types of subunits: a "monomeric" chain (chain I) and a disulfide bonded "trimer" of chains IIA, IIB and IIC. We have reported the complete amino acid sequences of all four chains (1) and proposed a "192-chain" model for the formation of Tylorrhynchus hemoglobin as follows (2):

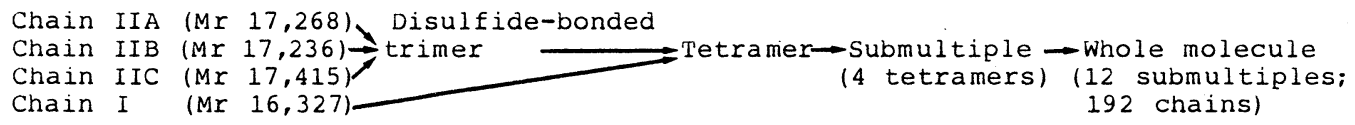

The sites of disulfide bonds in the "trimer" have now been determined. In the trimer, there are two inter-chain disulfide bonds between chains IIA and IIC, and IIB and IIC, respectively. In addition, each of the four chains, I, IIA, IIB, IIC, has an intra-chain disulfide bond. Thus, according to our "192 chain" model (2) there are 288 disulfide bonds in Tylorrhynchus hemoglobin, that is, 96 inter-chain bonds and 192 intra-chain bonds. Digital image processing of scanning transmission electron micrographs of negatively stained Tylorrhynchus hemoglobin indicated dimensions of $28 \times 18 \mathrm{~nm}$.

1. Suzuki, T. and Gotoh, T. (1986) J. Biol. Chem. 261,9257-9267.

2. Suzuki, T. and Gotoh, T. (1986) J. Mol. Biol. 190,119-123.

The existing mode of retinal and 3-dehydroretinal in the eggs of Xenopus laevis. Takaharu SEKI, Masami AZUMA, Shigechika FUJISHITA and Toshiaki IRIE

Dept. Health Sci., Osaka Kyoiku Univ. and "Meijôgakuin Highschool.

Eggs of Xenopus laevis contain nearly equal amount of retinal and 3-dehydroretinal, at the total amount about $50 \mathrm{pmol} / \mathrm{egg}$, but substantially no other retinoids and carotenoids $(1,2)$. During the development of the egg, the amount of both retinals, which are predominantly all-trans, decreases and that of the corresponding retinyl esters increases until stage 43. Azuma et al. (3) have shown that 11 -cis retinal and 3-dehydroretinal began to appear in the tadpole eye at the stage 40 , and that these retinals functioned as the chromophore of visual pigments in the eye at the stage $42 / 43$. As the tadpoles had been reared without feeding, the retinals contained in the eggs must be original precursors of the chromophore retinals. The existing mode of the retinals in the xenopus eggs was then examined as follows.

I) A homogenate of the ovulated eggs in $20 \mathrm{mM}$ Tris/HCl buffer (pH 7.4) was centrifuged at $12,000 \mathrm{rpm}$ for $20 \mathrm{~min}$; all of the retinals was detected in the precipitated fraction by an analysis using HPLC.

II) The precipitate was solubilized in the Tris buffer containing $400 \mathrm{mM} \mathrm{NaCl}$; all of the retinals was detected in the supernatant fraction after the centrifugation at $12,000 \mathrm{rpm}$ for $20 \mathrm{~min}$.

III) The substance containing retinals precipitated completely in the 658 sat. ammonium sulfate solution.

IV) After treatment of the retinal-complex with sodium borohydride, no retinoid was extracted by organic solvents, but the fluorescence and absorption of retinyl product were observed in the water layer including precipitates.

These results strongly suggest that the retinals are bound to the protein moiety of lipovitellin, a lipoprotein contained in the yolk of the amphibian yolk platelet, by the Schiff base linkage.

References:

1) Seki,T., Azuma,M. and Fujishita,S. (1986) Dôbutsu Seiri, $3,156$.

2) Seki,T., Fujishita,S., Azuma, M. and Suzuki,T. (1987) Zool. Sci., 4, 475-481.

3) Azuma,M., Seki,T. and Fujishita,S. (1988) Vision Res., in press. 
Actions of Mytilus inhibitory peptides on molluscan muscles. Tatsumi HIRATA, Ichiro KUBOTA*, Norio IWASAWA**, YukO FUJISAWA and Yojiro MUNEOKA Fac. Integrated Arts and Sciences, Hiroshima Univ., *Suntory Inst. Biomed. Res., and ${ }^{*}$ Suntory Inst.Fundment. Res.

Hirata et al. (1987a, 1988) have isolated one heptapeptide and two congeneric hexapeptides from the pedal ganglia of Mytilus edulis. The heptapeptide showed relaxing action on catch tension of the anterior byssus retractor muscle (ABRM) of the mussel, hence it was termed catch-relaxing peptide (CARP). The congeneric hexapeptides showed inhibitory action on phasic contraction of the ABRM in response to repetitive electrical pulses of stimulation, and they were termed Mytilus inhibitory peptides (MIPs, Ser2-MIP and Ala2-MIP). Although the actions of CARP on other molluscan muscles have been reported (Hirata et al., 1987b,c), those of MIPs have not yet been reported. In the present study, we examined the actions of MIPs on contractions of the ABRM of Mytilus and also on contractions of various other molluscan muscles. In the ABRM of Mytilus, MIPs inhibited not only phasic contraction but also contractions in response to $\bar{A} C h$, FMRFamide and caffeine. In contrast to CARP, however, the peptides did not affect catch tension. The peptides did not also affect catch-relaxing effects of serotonin, dopamine and CARP. In the pedal retractor muscle of Mytilus, contractions in response to repetitive electrical pulses and $\mathrm{ACh}$ were inhibited by MIPs. The cardiac activity of Meretrix lusoria was effectively inhibited by MIPs. The cardiac activity of Tapes japonica was inhibited by low dose $\left(10^{-7} \mathrm{M}\right)$ of MIPs, but it was accelerated by high dose $\left(10^{-} 5 \mathrm{M}\right)$. Twitch and tetanic contractions of the penis retractor muscle of Achatina fulica were also inhibited by MIPs. The present experiments have shown that MIPs are capable of inhibiting many molluscan muscles. MIPs and their analogues may be widely distributed in Mollusca. Recently, Fujisawa obtained some species with biological activity from the gel-filtrated acetone extracts of the ABRM. One of them showed MIP-like activity on the ABRM, suggesting that MIPs are physiologically involved in the regulation of function of the muscle as neuromediators. (1) Tatsumi Hirata et al. (1987a) Brain Res. 422, 374-376. (2) Tatsumi Hirata and Yojiro Muneoka (1987b) zool. Sci. 4, 987. (3) Tatsumi Hirata et al. (1987c) Zool. Sci. 4, 987. (4) Tatsumi Hirata et al. (1988) Biochem. Biophys. Res. Commun. (in press).

Hair sensilla on the genitalia of the male cricket and central projection of their primary afferent nerves. Chizu OHTA and Masaki SAKAI; Dept. Biol., Fac. Sci. , Okayama Univ.

The genitalia of the male cricket Gryllus bimaculatus comprises a sclerotized clasper, an epiphallic ventral lobe, a spermatophore sac and a water pillow-like membraneous structure lined with muscle strands. The clasper can be divided into three regions; medial process, lateral process and ectoparamere. About seventy hairs of the bristle type (.10um; 3-4um) were found on the medial process and 80 hairs $(, 100-200 u m ;, 4-5 u m)$ on the lateral process. The tips of both the processes bore larger hairs extending posteriorly. Thirty small hairs in cone shape (, 3-4um;,2um) were found in the caldera-shaped base on each of the proximal ectoparamere. Behavioral study indicated that those hairs on the epiphallus could be stimulated successively during copulation in contact with the subgenital plate and copulatory papilla of the female which in turn initiated pulling down the subgental plate, the expansion of both the ventral lobe and the water pillow-like structure, and the contraction of the spermatophore sac. The nickel filling through the cut end of the hairs revealed that the arborization of the primary afferent afferent terminals were almost the same in the $8 \mathrm{th}$ and $9 \mathrm{th}$ segments of the terminal abdominal ganglion. The epiphallic ventral lobe had totally 360 small hairs (,6-7um;, 3um) on the lateral and ventral surface but no hairs on the inside region where the ampula of a spermatophore was held. Each hair was situated on the top of an inverted cup-shaped basal structure sorrounded by many granular cells. Finally no hair sensilla was found on the water pillow-like structure continuous to the floor of the pouch. 
Morphology and distribution of the sensory hairs on the crayfish cephalothorax during development. Atsushi CHIBA, Masamitsu HAMAGUCHI, Masaaki KOSAKA and Shiko CHICHIBU. Dept. Physiol., Kinki Univ. Sch. Med.

Immature crayfish shows escape behaviours for stronger stimuli. As animals grow up, behaviour pattern changes from the escape to the defense. In order to correlate phenomena in the receptor levels with the development, the morphology and the sensory hair distribution on the cephalothorax were observed.

Twenty-three crayfish(Procambarus clarkii) ranging the body size from 4 to $52 \mathrm{~mm}$ were examined with a SEM. Crayfish were fixed with glutaraldehyde and paraformaldehyde, and dehydrated through a graded series of aceton. Then, critical point drying was adopted, coated with gold, and examined with a Hitachi S-450. Reconstruction of changes was made with sequences of SEM photographs.

The number and kind of the sensory hairs increased with the development. The sensory hairs on the crayfish of intermediate sizes were $5 \mu \mathrm{m}$ long in $5 \mathrm{~mm}$ body length, and $43 \mu \mathrm{m}$ long in $52 \mathrm{~mm}$ specimen. Number of the sensory hairs, however, reached to a saturation, which was about $1.1 \times 10^{3}$ hairs on a $35 \mathrm{~mm}$ body length. Sensory hairs were divided into either cuspidate or pappose/plumose types under body length of $20 \mu \mathrm{m}$. When the body length increased over $20 \mathrm{~mm}$, a small round eminence became distinct on the caudal neighbour of the hair pit, respectively. The combination consisted of more than two hairs increased on the ventral carapace. Taking as the density of the sensory hairs as $60 / \mathrm{mm}^{2}$ in $7 \mathrm{~mm}$ specimen, the density decreased with the development, and reduced to $20 / \mathrm{mm}^{2}$ in $52 \mathrm{~mm}$ specimen. Though the sensory hairs around the eye stalk did not change its number.

The above results suggested that the sensory hairs on the cephalothorax were opted for collecting informations both quality and quantity regarding the external stimuli significant for their lives.

Involvement of calcium in adaptation mechanism of sugar taste receptor cell of $\mathrm{fly}$ Mamiko Ozaki and Taisaku Amakawa. Dept. Biol., College. Gen. Education, Kobe Univ.

Previously, we reported that protein kinase C (calcium- and phospholipiddependent protein kinase) may be deeply concerned with taste adaptation in sugar receptor of fly. This raised an interest in contribution of calcium to the adaptation.

When the receptor cell was stimulated with sucrose solution containing $\mathrm{CaCl}_{2}$ ' its response was almost same as that to plain sucrose stimulation as long as the concentration of $\mathrm{CaCl}_{2}$ was less than $2 \mathrm{mM}$. On the other hand, the receptor cell, when pretreated with a calcium ionophore, A23187, showed strong adaptation in calcium dependent manner. Thus, the concentration of intracellular calcium was suggested to regulate the adaptation. However, calcium influx through ion cannel during cell excitation seemed not to promote adaptation.

Then, we gave inositol trisphosphate ( $\left.\mathrm{IP}_{3}\right)$ into the cell and again observed strong adaptation. IP likely to release calcium to regulate adaptation from intracellular reservoir. Since $I_{3}$ and diacylglycerol(DG) were generated by phosphatidylinositol bisphosphate (PIP,) degradation, this taste reception may couple with inositol phospholipid metabolism. Calcium released by IP and DG were well known as essential activators for protein kinase C. Thus, the present work appears to consistent with our previous report suggesting contribution of protein kinase $C$ to the adaptation mechanism in sugar taste receptor cell of fly. 
Turning reactions to light stimuli in the posterolateral eyes of the jumping spiders, Hasarius adansoni and plexippus paykulli. Shigeki YAMASHITA and Miki KOMIYA* Biol. Lab., Kyushu Inst. Design and *Dept. Biol., Fac. Sci., Kyushu Univ.

Jumping spiders reacted to the turning on and off of a single stationary spot of light placed in the visual field of the posterolateral eyes, with a accurate turn of the prosoma. Single off-stimulation of up to $7^{\circ}$ in spot size and single on-stimulation of up to $3^{\circ}$ are sufficient for the reaction. The rate of reactions to single light stimuli decreased with growing size of a spot, with decreasing intensity of light, with decreasing rate of change in intensity, and with decreasing duration of shadow or light.

The rate of reaction to two sequential off-stimuli from two spots $\left(1^{\circ}\right.$ aperture each, center-to-center distance of $1^{\circ}$, increased markedly as compared with that to single off-stimuli from one spot. For movement detection, both the change in intensity for each receptor and the temporal interval between two stimuli played important roles. The rate of reaction to simultaneous off-stimuli from two spots decreased as compared with that to single off-stimuli from one spot. The inhibitory effect was at maximum at a distance of $5^{\circ}$. The rate of reaction to sequential on-stimuli increased slightly as compared with that to single stimuli. In addition, simultaneous on-stimuli at $3-7^{\circ}$ spacing also enhance the reaction.

A possible role of cyclic GMP in transduction mechanism of sugar taste receptor cell of fly, Phormia regina. Taisaku AMAKAWA and Mamiko OZAKI, Dept. Biol., Col. Gen. Educ.,Kobe Univ.

In some vertebrate sensory cells as rod cell,olfactory cell or taste cell, cyclic GMP and/or cyclic AMP are shown to mediate information from receptor molecule to ion channel. We studied the effects of dibutyryl cyclic GMP (dbcGMP) and other nucleotides on sugar receptor excitation of fly, Phormia regina. The tip recording method (Hodgson et al. 1955) and the side-wall recording method (Morita and Yamashita,1959) were employed. When $20 \mathrm{mM}$ dbcGMP, which was known to permeate cell membrane, was given to the tip of the sensillum, impulses of large size were evoked. Other nucleotides also evoked the same impulses but their frequency was less than $1 / 3$ of that of dbcGMP. From the results obtained that size of impulses corresponded to that of sugar receptor cell, and that dbcGMP given to labellum induced proboscis extension reflex (feeding response), the impulses were identified as those from sugar receptor cell. Application of dbcGMP to the chemosensillum brought about some characteristics different from sugar stimulation. For instance, extremely low adaptation was observed. The impulse discharge was seen even after removal of the $20 \mathrm{mM}$ dbcGMP mixed with cyclic nucleotide esterase $(2.5 \sim 10 \mathrm{mM}$ isobutylmethylxanthine or $10 \mathrm{mM}$ theophyline). We speculate that the prolonged life of cyclicGMP by inhibition of the esterase held ion channel open for a while after removal of dbcGMP solution from the sensillum. The results obtained by us suggest that cyclic GMP regulates ion channels of fly sugar-receptor membrane, directly or indirectly. 
Contribution of the external divalent cations on the time dependent decay of the olfactory response and of the cyclic AMP-induced current in solitary olfactory receptor cells from newt. Takash1 KURAHASHI and Tatsuaki SHIBUYA Inst. Biol. Sc1. Tsukuba Univ.

It is well known that the olfactory receptor cells adapt to the prolonged odor stimulation. This study was undertaken to know the mechanisms of the adaptive properties of the olfactory response. The olfactory cells isolated from newt were used to record an olfactory response.

The olfactory response evoked by some odorants ( $n$-amyl acetate, D-limonene) showed phasic depolarization to a prolonged application $(3-9 \mathrm{sec})$. It is already known that the depolarizing response arose from the activation of a cationic conductance. Under the voltage clamp condition, the odorant-activated conductance showed time dependent inactivation with similar time course to the olfactory response. Furthermore, the amplitude of the odorant-induced current reduced by the preceding odor pulse. These results suggested that the adaptive properties of the olfactory response is due to the time dependent inactivation of the transduction current.

We further found that such a time dependent inactivation of transduction current depended on the external divalent cation concentration. When the external divalent cation was extruded almost completely, the odorant-activated conductance did not inactivate during the stimulation.

Similar time course of a current was observed, when cAMP, which is a possible candidate for the transduction mediator, was diffused into the cell from recording pipette. In addition, the inactivation process of the current also required the external divalent cation, either $\mathrm{Ca}$ or $\mathrm{Mg}$.

Assuming from the time dependency of the decay, the divalent cation is reasonably considered to affect to the chemotransduction machinery from intraceliuler side passing the membrane through the transduction conductance itself.

Degeneration of flight muscles in the cricket Gryllus bimaculatus. Sakiko Shiga, Katsunori Yamada, Tsuneo Yamaguchi, and Kohji Yasuyama* Dept. Biol., Fac. Sci., Okayama Univ. and *Dept. Biol., Kawasaki Med. Coll.

In both sexes, the metathoracic dorsal longitudinal flight muscle (M112a) grows in size for $72 \mathrm{hr}$ following the imaginal molt, and then it degenerates rapidly (Yamaguchi et al., 1987). It was evident that during the degeneration of M112a it shows the change in appearance from orange to white, and this change is accompanied with the decrease in both mitochondrial content and cytochrome $c$. Disappearance of birefringence of $\mathrm{M} 112 \mathrm{a}$ was found to start always from its ventral part within $72 \mathrm{hr}$ following the final molt and spread over the whole muscle, suggesting that the muscle fibers in the ventral part of M112a precede those in the dorsal part in the degeneration. As far as birefringence of degenerating muscle remained visible, its electrical and mechanical responses to stimuli were basically identical to those of the M112a in which any degenerative change was not observed. the degeneration of M112a was unaffected by severance of the motoneurons innervating the $M 112 \mathrm{a}$ and also by $\mathrm{f} i \mathrm{xation}$ of the wing to prevent its movement. Decapitation within $48 \mathrm{hr}$ following the imaginal molt, however, suppressed the degeneration of muscles, though the decapitation after more than $72 \mathrm{hr}$ following the imaginal molt had no effect on the degeneration. Results suggest the presence of a strong correlation between the degeneration of metathoracic dorsal longitudinal flight muscle and hormonal factors from the head region. 
Analysis of the chromophore isomerization mechanism in rhodopsin. Tôru łOSHIZAWA, Yoshinori SHICHIDA, Katşuki NAKAMURA, Robert S. H. Liu*, Alfred E. ASATO , Marlene DENNY $^{\star}$ and Achla TREHAN ${ }^{*}$. Dept. Biophys., Fac. Sci., Kyoto Univ. and Dept. Chem., Univ. Hawaii.

Since the chromophore of rhodopsin is photo-isomerized in such a restricted space as the chromophore binding site of opsin, two possible photoisomerization models of the chromophore other than one-photon-one-double-bond isomerization model (Model 1) have been proposed; one is so called "Bicycle pedal" model (one-photontwo-double-bond isomerization model, Model 2, Warshel, 1976) and the other is "Hula twist" model (Concerted isomerization of one double bond with neighboring single bond, Model 3, Liu and Asato, 1985). Models 2 and 3 are suitable for the isomerization in the specially limited structure. In order to verify these nodels, some rhodopsin analogs were used and their photochemical reactions were investigated. 9,13-Dicis rhodopsin was reported to be converted directly to all-trans form upon irradiation (Crouch et al., 1975). It seems to support the one-photon-twodouble-bond isomerization model (Model 2). However, the present investigation revealed that incubation of 9,13-dicis retinal and opsin yielded a mixture of 9,13dicis rhodopsin as a minor component and 9-cis rhodopsin as a major component and irradiation of 9,13-dicis rhodopsin converted the chromophore to 13-cis form. These results clearly demonstrated that one-photon-one-double-bond isomerization process took place in 9,13-dicis rhodopsin.

According to the Model 3 , the structure of chromophore of bathorhodopsin was suggested to be in 10-s-cis, all-trans form (Liu and Asato, 1985). If so, bathointermediate of the rhodopsin analog having 10-s-trans-locked retinal as its chromophore should not be produced. Low temperature spectrophotometry combined with chromophore extraction experiments using the rhodopsin analog revealed that batho-intermediate having all-trans chromophore was definitely produced. Therefore, the chromophore of bathorhodopsin should not be in a 10-s-cis conformation.

All these results supported the view that the chromophore of rhodopsin is isomerized according to one-photon-one-double-bond isomerization model (Model 1 ).

The in vivo synthesis of 11 -cis retinoid in the mouse. Tatsuo suzuKI, Yoshiaki IMOTO and Sadao MIYATA Dept. Pharmacol., Hyogo Coll. Med.

We have already reported that 11-cis dehydroretinal accumulated in the eye of the vitamin A-depleted mouse after the injection of all-trans dehydroretinol (1). In the present study, we determined the spectral sensitivity curve of ERG and the absorption spectrum of visual pigment in the mouse injected with dehydroretinol. Absorption maximum of the visual pigment was located at the wavelength about $20 \mathrm{~nm}$ longer than that in the retinol-injected animal. The spectral sensitivity curve was consistent with the absorption spectrum of the visual pigment. These results show that the dehydroretinol is utilized by the mouse for the chromophore of visual pigment without conversion into retinol.

The visual pigment was produced when the animal was injected with all-trans dehydroretinol and then kept in constant darkness. The process of pigment synthesis was very slow, with a half-time about 4 days. About a half of normal level of rhodopsin was generated when the retina homogenate of the vitamin Adepleted mouse was incubated with 11 -cis retinal. These results indicate that the isomerization of retinoid is the limiting process of the visual pigment synthesis. The pigment synthesis was much slower in the cyclic light and dark conditions (LD) than in constant darkness. A considerable amount of free opsin was detected in the eye of the LD animal after overnight dark-adaptation. The reaction of retinoid isomerization is too slow to regenerate all pigments during the overnight dark-adaptation. It was reported that the outer segments of the rat photoreceptor cells were lost in the extreme deficiency of vitamin A (2). In our vitamin A-depleted mouse, a nearly normal structure of the outer segment layer was observed with microscopy in the retina section. It was also reported that the length of rod outer segment of the albino rat changed in response to the light intensity (3). It remains to be examined whether some morphological changes are involved in the slow process of pigment synthesis in the albino mouse.

(1) Suzuki T. and Miyata S. (1988) Exp. Eye Res., 46, 161-172.

(2) Dowling J.E. and Wald G. (1958) Proc. Nat. Acad.'Sci., 44, 648-661.

(3) Penn S.P. and Williams T.P. (1986) Exp. Eye Res., 43, 915-928. 
The diurnal change of the sensitivity and the two-dimensional acceptance angle in the retinu1a cell of Ligia exotica.

Takahiko HARIYAMA and Yasuo TSUKAHARA Research Center for Applied Information Sciences, Tohoku Univ.

Katahira 2 -chome, Sendai 980 , Japan

Both the structure of the retinula cells and the ERG amplitude have been known to change diurnally in Ligia exotica, irregular arrangement of microvilli and increase in the ERG amplitude (At midnight the sensitivity recorded by ERG is more than 10 times as large as that of the day) were observed. Intracellular recordings from the retinula cells showed no difference in the slope of $V-10 g$ I curve but the sensitivity at midnight is less than 1.5 times as large as that of the day. The iso-two dimensional angular sensitivity curves of single units recorded. at night were apparently irregular which seems to correspond the irregular arrangement of microvilli. The angular sensitivity fields are irregular at night and regular at day. Both during the night and the during the day, however, the fields were with a width of ca.2 at the $50 \%$ leve1. Morphologically, we have not observed the pigment granule migration arround the dioptric apparutus, and the lens system of the dioptric apparatus is a kind of afocal telescope. Diurnal changes in the total amount of retinal isomers were studies by high performance liquid chromatography (HPLC) in the retina (Hariyama \& Tsukahara 1988). During the night, the 11-cis form of retinal oxime is three times as large as that during the day. The concentration of rhodopsin does not change during day and night, because the volume of the rhabdom during night is three times bigger than that during night (Hariyama et a1.1986). The tips of rhabdom were penetrated into the end of the crystalline cone during the night, however the tips were adjacent to the crystalline cone end during the day. The volume change of rhabdom seems not to occur for the increase of sensitivity during the night.

Analyses of synaptic potentials of tectal neurons of the frog elicited by "on-off" and "off" retinal fibers. Nobuyoshi MATSUMOTO, Atsushi TAMADA and Yukako TADA, Department of Biophysical Engineering, Faculty of Engineering Science, Osaka University, Toyonaka, Osaka 560, Japan.

Retinal ganglion cells are divided into 4 classes according to their response properties. Tectal neurons are also classified into several types according to extracellular or intracellular recordings. The purpose of this experiment is to elucidate synaptic organization and present spatial receptive field structure of tectal neurons.

The optic tectum was exposed under MS222 anesthesia and immobilized with succinylcholine. A single unit of "on-off" or "off" response was isolated at their terminals using a tungsten electrode, and intracellular potentials were simultaneously recorded from a tectal neuron with another microelectrode. Pulse-analogue correlation method was applied, and resultant postsynaptic potentials were examined. The result shows that "on-off" fibers monosynaptically excite E-E type (EPSP at "on" and "off" of light) and EI-EI type (EPSP-IPSP at "on" and "off") tectal neurons. "Off" fibers also monosynaptically excite IE-IE type (IPSP-EPSP at "on" and "off") neurons.

Unless we use 3 electrodes, we cannot analyze synaptic connections of 2 fiber classes onto a single tectal neuron. Based on the latencies of "on-off" elicited and "off" elicited responses, we could successfully differentiate the synaptic potentials by "on-off" fibers by those from "off" fibers. One example in the present preliminary result indicates that an EI-EI type tectal neuron has "on-off"induced excitation and surrounding inhibition, and this area is surrounded by a huge "off"-induced inhibitory area, which exeeds 90 degrees in diameter. Such receptive field organization could not be derived from the ordinary examination with moving stimuli.

Reference

Nagano K, Li Q-L, Tamada A, Matsumoto N, Correlation of postsynaptic potentials of tectal neurons of the frog with simultaneously recorded impulses from terminals of retinotectal afferents. Exp Brain Res 70 (1988) 439-442. 
The seasonal changes of the emergence rhythm of Dimorphostylis asiatica Zimmer (Crustacea, Cumacea). Tadashi AKIYAMA, Kohzoh OHTSU and Masao YOSHIDA Ushimado Marine Lab., Fac. Sci., Okayama Univ.

Marine crustacean Dimorphostylis asiatica Zimmer emerge nocturnally from their daytime habitat, which is the muddy sand at some depth. Overnight collections done bimonthly for 15-40 consecutive days in each series revealed unique seasonal changes in the emergence pattern.

Spring (March-May): Times of the nocturnal emergence delayed day by day keeping good correlation with times of nocturnal high waters, i.e., D. asiatica showed clear tidal periodic activity (tidal PA). The daily periodic activity (daily PA) appeared at both dusk and dawn but only transiently when peak activities jump from dawn to dusk. In May, the appearance of the tidal PA was accompanied by increase in the number of animals captured.

Summer (July): The emergence pattern was modified with the pace of the lunar cycle. From the full moon to the first quarter of the moon, the daily PA was dominant, and around the full moon, the tidal PA. The number of animals captured in each overnight collection also fluctuated with the pace of the lunar cycle and reached the maximum around the full moon, where dominant tidal PA was seen.

Autumn (September-November): D. asiatica emerged dominantly around the sunset throughout this season, i.e., D. asiatica showed dominant daily PA with extremely weak tidal PA.

Winter (January): In the earlier half of January, D. asiatica showed dominant daily PA around the sunset and relatively weak tidal PA. In the later half of January, however, the tidal PA became dominant, suggesting return to the spring pattern.

Facilitatory factors on spermatophore protrusion in the male cricket. Takahiro OTSUBO and Masaki SAKAI Dept. Biol., Fac. Sci.. Okayama Univ.

The spermatophore protrusion (SPP) in the male cricket Gryllus bimaculatas starts usually several to $30 \mathrm{~min}$ after the end of copulation (CP). The interval (CP-SPP) between CP and SPP varies in contrast to the interval (about $60 \mathrm{~min}$ ) from SPP to calling song (CS). We examined what factors were involved in determining the CP-SPP. Experiments were carried out in a $200 \mathrm{ml}$ beaker under a light condition at $27^{\circ} \mathrm{C}$. The median of a frequency histogram on CP-SPP was used as a representative for an experimental group.

1) The higher temperature $\left(33^{\circ} \mathrm{C}\right)$ than the standard $\left(27^{\circ} \mathrm{C}, 6 \mathrm{~min}\right)$ did not affect CP-SPP $(6 \mathrm{~min})$ while the lower temperature $\left(20^{\circ} \mathrm{C}\right)$ increased $\mathrm{CP}-\mathrm{SPP}(12$ min). 2) CP-SPP was almost the same at different levels of environmental light intensity; $4.5 \mathrm{~min}$ at $\langle 1 \mathrm{lux}, 6 \mathrm{~min}$ at $301 \mathrm{ux}$ and $4.5 \mathrm{~min}$ at $3001 \mathrm{ux}$. 3) CP-SPP increased considerably $(>60 \mathrm{~min})$ in the condition in which the male was left alone after copulation. The similar results were obtained when the antenna and palpi were surgically removed or when the bodily contact was prevented by hanging the female above the male. 4) When males were kept separated from females for $24 \mathrm{~h}$ before experiments, CP-SPp decreased to $4 \mathrm{~min}$ even under the separated condition after copulation. 5) The removal of the accessory gland slightly delayed the start of the spermatophore protrusion $(12.5 \mathrm{~min})$. However, it was shortened to $7 \mathrm{~min}$ after the separation of $24 \mathrm{~h}$ from females, indicating that the sexual deprivation effect was not caused by a decrease in activity of the accessory gland. 6) The CP-SPP $(7 \mathrm{~min})$ of the testisless was not different from that $(6 \mathrm{~min})$ of the intact males.

we concluded that spermatophore protrusion was facilltated by chemical substances of the female via contact and by deprivation of the copulation. 
Possible involvement of GABAergic neurons in the diapause hormone secretion of the silkworm, Bombyx mori. Isamu SHIMIZU, Tomohiko MATSUI and Kou HASEGAWA:Research Section of Environ. Biol., Labo. for Plant Ecological Studies, Fac. Sci., Kyoto Univ. Kyoto 606

The silkworm is a typical insect species of embryonic diapause and the diapause is determined by various environmental conditions which the mother moths have experienced during their embryonic and larval stages. The diapause hormone(a peptide hormone) is secreted from neurosecretory cells located in the subesophageal ganglion (SG) of the diapause eggs producer during the pupal stage, acting on the developing ovaries and produces diapause eggs. While in non-diapause producer the secretion is blocked and the eggs laid become non-diapause. Some pieces of evidence obtained by surgical experiments strongly suggested that brain ( $\mathrm{Br}$ ) of the silkworm controls the secretion from $\mathrm{Sg}$ through subesophageal connective. However, the physiological mechanism governing the diapause hormone secretion has not been revealed yet. To see an involvement of GABAergic neuron in the diapause hormone secretion picrotoxin (PXT) and bicuculline, which block the GABA mediated synaptic inhibition, was injected into 4 day-old pupae of non-diapause producers. After adult ecdysis the diapause and non-diapause of eggs were examined by Ehrich's diazo reaction. In non-injected and saline-injected controls all female moths laid only non-diapause eggs. on the other hand, the potent blockers of GABAergic neuron induced diapause eggs. Injection of PXT following removal of SG of the hormone source, did not produce diapause eggs:these agents act on the central nervous system of $S G-B r$. Injection of GABA into diapuae eggs producer pupae produced some moths having non-diapause eggs. These pieces of evidence strongly suggest that GABAergic neurons function in the neuroendocrine control of the diapuse hormone in the silkworm.

Characteristics of the sodium channel current in embryonic rat heart cells.

Naoki HINO and Kenji KOIZUMI* Dept. Physiol. and Dept. Anat.*, Sch. Med., Juntendo Univ.

The excitabilty of the embryonic hearts appears with an increase in the resting potential. In 14-day rat embryo hearts, ventricular myocardium generates the sodium dependent action potentials from a low resting potential of around $-60 \mathrm{mV}$. This is in the range of the membrane potentials at which the sodium channel current( (INa) in adult myocardium is almost inactivated. This suggests that the voltage-dependence of $I_{\mathrm{Na}}$ kinetis in embryo is different from that in adult. We studied the kinetic properties of INa in the ventricular myocytes of rat (Sprague-Dawley) embryos, using the patch-pipette whole-cell clamp method. The internal and external solutions contained $\mathrm{Na}^{+}$at concentrations of $20 \mathrm{mM}$ and $135 \mathrm{mM}$, respectively.

$I_{\mathrm{Na}}$ elicited by depolarizing voltage steps from a holding potential of $-85 \mathrm{mV}$ had a threshold between -50 and $-45 \mathrm{mV}$ and reached a peak in less than $0.8 \mathrm{msec}$ over the voltage range from -20 to $+100 \mathrm{mV}$. This was completely blocked by $30 \mu \mathrm{m}$ tetrodotoxin. The amplitude of $I_{\mathrm{Na}}$ estimated as a difference between its peak and the steady-state current at $30 \mathrm{msec}$ was maximal at -20 to $-10 \mathrm{mV}$, the current density being $0.25 \mathrm{~mA} / \mathrm{cm}^{2}$ $(n=15)$. The reversal potential estimated from the current-voltage plots for INa during the voltage steps and for its tail currents was $+50 \mathrm{mV}$, which agreed with the value predicted by the Nernst equation. The voltage-dependence of steady-state inactivation was examined by applying conditioning prepulses before stepping to a depolarization to $-15 \mathrm{mV}$. INa was diminished on prepulses positive to $-75 \mathrm{mV}$ and was blocked at -40 to $-35 \mathrm{mV}$. The steady-state activation and inactivation parameters of $I_{N a}\left(m_{\infty}\right.$ and $\left.h_{\infty}\right)$ were calculated according to the $m 3 h$ model. Half activation and inactivation potentials were in the ranges of -36 to $-33 \mathrm{mV}$ and -61 to $-49 \mathrm{mV}$, respectively. These values were $15 \sim 40 \mathrm{mV}$ more positive than those obtained from adult cells. INa recovery from inactivation was measured applying double pulses to $-5 \mathrm{mV}$ with variable pulse intervals. Half recovery time was $2 \mathrm{msec}$ and $8 \mathrm{msec}$ when the holding potentials were -95 and $-75 \mathrm{mV}$, respectively. These values were $20-f o l d$ faster than those reported on adult cells, reflecting the positive shift in the voltage-dependence of inactivation of $\mathrm{I}_{\mathrm{Na}}$. 
Effects of tetanic stimulation on acetylcholine quanta at the frog neuromuscular junction. Hiroshi KITA Dept. of Physiol., Kawasaki Med. Sch.

Using sciatic nerve-sartorius muscle preparations of the frog, the effects of tetanic stimulation of the nerve on the frequency and amplitude of intracellularly recorded miniature end-plate potentials (MEPPs) were investigated. (1) After treatment of the preparation with $2 \mathrm{M}$ formamide to eliminate muscle contraction, supramaximal stimulation at $10 \mathrm{~Hz}$ for $5 \mathrm{~min}$ in normal saline solution produced, in a typical example, a 3.6 -fold increase in the frequency which subsequently declined roughly exponentially toward the initial level. On the other hand, the amplitude fell to a level which was 0.37 times that of the control immediately after the tetanus and then recovered gradually toward the pre-tetanus level. However, it did not return to that level even after $45 \mathrm{~min}$. After stimulation, the values of the coefficient of variation (c.v.) of the MEPP amplitudes were above the control value for more than $40 \mathrm{~min}$. (2) The same experiments were repeated in the presence of AH5183, which is known to inhibit the active transport of acetylcholine into synaptic vesicles. Following tetanic stimulation the decreased amplitude first showed a tendency to recover to the initial level, but then rather decreased slightly and stayed at that level even after the drug was removed. The values of the c.v. were below the control after the tetanus and, in a typical case, did not return to it even after about $1 \mathrm{hr}$. (3) To record MEPPs during tetanic stimulation, the concentration of $\mathrm{Ca}^{2+}$ of the bathing solution was reduced to $0.1 \mathrm{mM}$ and that of $\mathrm{Mg} 2+$ was raised to $3 \mathrm{mM}$, and the frequency of tetanus was changed to $2 \mathrm{~Hz}$ with its duration unchanged. Despite increases in MEPP frequency during and after stimulation, the MEPP amplitude was almost the same as that of the control throughout these periods which extended to 20 min after the tetanus. The values of the c.v. of the amplitudes were higher during the tetanus and were about the same as that of the control after it. (4) For the same purpose, stimulation at $10 \mathrm{~Hz}$ for 5 min was applied in a solution whose only divalent cation was $2.5 \mathrm{mM} \mathrm{Mn} \mathrm{M}^{2+}$. In one example, the tetanus increased the frequency 4.09-fold, but reduced the amplitude to a level slightly below that of the control. The lowered level remained unchanged until 30 min after the tetanus. The values of the c.v. of the amplitudes decreased below that of the control during the tetanus and recovered gradually.

Stretching is required for recovery of intramembrane particle density and afferent responses in sensory axon terminals of frog muscle spindle. Noriaki FUJITSUKA, Atsushi YOSHIMURA, Chie FUJITSUKA, Keisuke KAWAKAMI and Fumio ITO. Dep. of Physiol. Nagoya Univ. Sch. Med.

Recently we reported that the population density of intramembrane particles fell to zero within a week after denervation by nerve crush, and then increased to $120 \%$ of control at 2 months in the course of reinnervation, while a supersensitivity to stretch was observed. The changes of particle density during nerve regeneration led us to further investigation of factors modifying particle density. This study is part of a histophysiological investigation on the regeneration of sensory axon terminal in frog muscle spindle after tenotomy with or without nerve crush. Adult frogs Rana catesbeiana were anesthetized. The left sciatic nerve was crushed at the dorsal part of the hip joint and the distal tendons of semitendinosus muscles in both legs were cut. The right semitendinosus muscle was used to observe the effects of tenotomy alone. After operation, the frogs were fed for 2 to 150 days. Intact animals were used as controls. Spindle axons isolated from semitendinosus muscles were set in an air-gap, and the afferent responses to ramp-and-hold stretch were recorded. After the physiological studies the spindles were freeze fractured for making replicas. Particle size and density were measured on electron micrographs (X 200,000).

The majority of intramembrane particles were associated with the protoplasmic face of axons. The particle density increased to more than twice the control value by 2 months after tenotomy and both static and dynamic responses were enhanced. Tenotomy with nerve crush resulted in a decrease of the particle density and disappeared within 8 days. By 2 months, a few muscle spindles had restored phasic responses during dynamic phase of stretch, but there was no static response during maintenance of stretch. No further recovery in the stretch responses was observed by 3 months following the operation. It is concluded that stretching the muscle after nerve crush is required for recovery of normal particle density and afferent responses. 
Nonspiking interneuron in the terminal abdominal ganglion of the cricket: thier morphology and responsiveness to wind stimulation. Yoshichika BABA, Kiyonori HIROTA and Tsuneo YAMAGUCHI, Dept. Biol., Fac. Sci., Okayama Univ.

In the terminal abdominal ganglion ( $T A G$ ) of the cricket (Gryllus bimaculatus), thirteen nonspiking interneurons (NL-1 - NL-13), which responded with graded potentials to wind stimulation of the cerci, were identified according to their neural architecture and response characteristics.

Among these nonspiking interneurons were nine bilateral neurons (NL-1 - NL9 ), of which the dendrite and neurons and four unilateral (NL-10 - NL-13), of which the whole structures were located within either half. Apart from NL-3, all the interneurons exhibited the directional sensitivity; both the polarity and amplitude of graded responses were determined by the directions of wind.

In NL-2 which has been encountered most frequently, the response characteristics of each neurite were examined by marking the recording site with ethidium bromide. It was evident that the anterior and posterior neurites responded with depolarizing potentials to the wind from the directions contralateral to the soma and that from the ipsilateral directions, respectively.

The roles of these nonspiking interneurons are supposed to play as integrators or relay neurons in the sensory information pathways from the cercal neurons to higher order neurons.

Stretch receptor organs of the pill bug( Armadilldium vulgare): Morphology and Physlology. Akiyoshi Ni ida, Kouichi Sadakane and Tuneo Yamaguchi, Dept. of Biol., Fac. of Sci., Okayama Unv.

The presence of stretch receptor organs (SRO) of the terrestrial crustacean was demonstrated by staining with metylen-blue, back-filling and electrical recording. These organs occurred on either side of the midline in each segment (2nd to $7 \mathrm{th}$ ). On each side of the 2nd thoracic segment there is only one stretch receptor organ which has no receptor muscles: a stem dendrite leaving the cell body of a receptor neuron runs in closely parallel with the medial longtudinal muscle, attaching to the anterior tergal ridge of the 1st segment, and another attachment is seen in the articular menbrane of the ist segment. While in the 3rd the SRO comprises two components: medial SRO and lateral SRO. The medial receptor muscle has its affix within its own segment but lateral one runs through two segments, where these two receptor muscles parallel about $300 \mu \mathrm{m}$ apart. In the fourth segment and in the subsequent segment such parallel receptor muscles are seen to fuse and thus the same also occurs in the cell body of the receptor neuron.

The stretch receptor neurons on each segment are projected to the relevant thoracic ganglia via the 3rd nerve root unlike that of the cryfish.

The stretch receptor neurons ( 2 nd to $7 \mathrm{th}$ ) showed tonic discharge to the muscle stretch. This indictes that the stretch receptor neurons are tonic type. Furthermore, GABA effect on the 2 nd $s$ tretch receptor neuron was studied. Application of $4 \times 10^{-4} M$ GABA suppressed discharge induced by a steady stretch but impulses never ceased. Replacing the solution by normal saline restored its activity but subsequent application of GABA at the same concentration occurred no applicable change in a discharge frequency. 
Changes in orientations of abdominal sensory hairs with the development in the crayfish. Masamitsu HAMAGUCHI, Masaaki KOSAKA, Atsushi CHIBA and Shiko CHICHIBU. Dept. Physiol., Kinki Univ. Sch. Med.

Sensory hairs on the abdominal carapace of the cryfish began to develop immediately after hatching. Hair number, shape and distribution change with developmental stages. SEM observations were made on juvenile crayfish (Procambarus clarkii) of both sexes, and of 3 to $10 \mathrm{~mm}$ body length specimens. Specimens were pre-fixed with a mixture of $4 \%$ paraformaldehyde and $4 \%$ glutaralde hyde solutions for 2 hours. They were routinely processed for critical point dehydration with aceton series. Preparations were coated with gold, and obser ved with a HITACHI S-450 SEM. These photographs were used for serial reconstruction. At the 1st abdominal segment the number of sensory hairs increased in proportion to body length with the development. The density of sensory hairs per unit surface area decreased progressively with the developement. This relation was also observed in the 2 nd - 5 th abdominal segments. The abdominal sensory hairs were of two different types. One is the branched hair and another is the unbranched one. Branched hairs consisted the majority and the unbranched hairs were very few. The length of the hair in both types increased with the development. Crayfish smaller than $10 \mathrm{~mm}$ body length had branched hairs on all over the abdominal segments, and unbranched hairs were observed on limited particular region. Hairs on the dorsal surface of the 1st abdominal segment arranged in the right angle to the body axis, while hairs on the ventral fringe of the segment were forming circular arrangement. As these hairs were innervated by mechanosensitive neurons respectively, this organized orientation of hairs would be convenient to get successive and patterned informations from the environment as well as useful for the central processing of the incoming neural informations.

Responses to hygro-stimulation in the deutocerebrum of Periplaneta americana Fumio Yokohari Biological Lab., Fac. Sci., Fukuoka Univ. 814-01 Fukuoka

A good deal have been investigated about the structure and function of insect hygroreceptors. There have, however, been few data on characteristics of responses of the central nervous system to hygrostimulation on the antennal hygroreceptors in insects. We recorded impulse activities of deutocerebral neurons of the male cockroach, Periplaneta americana L., when the antennal hygroreceptors were exposed to humidity stimulation.

Sites of impulse recording: (see Figure)

Impu $\frac{1 s}{\mathrm{act} i \mathrm{ities}}$ responding to hygrostimulation were recorded within the centro-dorsal part of an ipsilateral deutocerebrum. Furthermore, excitatory responses to increase in humidity were recorded dorsally in the region and those to decrease in humidity were recorded ventrally in it.

Patterns of time courses of responses:

Several types of responses to increase of humidity were recorded; excitatory phasic tonic type, excitatory phasic type, inhibitory type, and inhibitory-excitatory type etc.

Stimulus intensity-response relationships:

Several types of stimulus-response curves were obtained. 1) The impulse frequency decreases with increase in humidity. 2) The impulse frequency increases with increase in humidity. 3) The impulse frequency attains to peak at intermediate humidity. 4) The impulse frequency slightly depended on change of humidity.

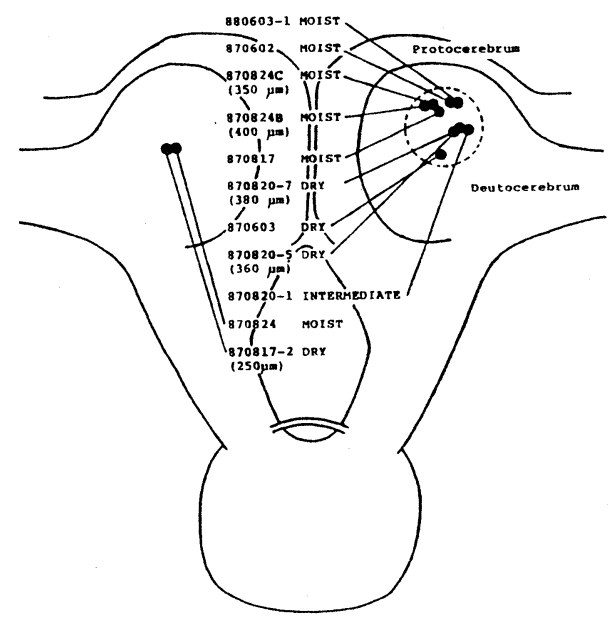


Immunohistochemistry with monoclonal antibodies against chicken iodopsin on the chicken and quail retinas. Tadashi OISHI, Tomoko HAYASHI, Yoshitaka FUKADA* and Toru YoshIzAWA Dept. Biol., Fac. Sci., Nara Women's Univ., and *Dept. Biophys., Fac. Sci., Kyoto Univ.

Immunohistochemical localization of iodopsin with chicken monoclonal antibodies was studied using Vectastain ABC system (Vecter) in the paraffin sections $(4 \mu \mathrm{m}$ thickness) of chicken (Gallus domesticus) and quail (Coturnix Coturnix japonica) retinas. We used four monoclonal antibodies (I1E9, I1ET2, I2F5 and I2F7) against chicken iodopsin and a polyclonal antibody of bovine rhodopsin. (1) Observations in normal chicken and quail retinas. All the monoclonal antibodies of iodopsin $(5-10 \mu \mathrm{g} / \mathrm{ml})$ stained the outer segments of double cones (both primary and accessory members) and some single cones, but they did not stain the outer segments of rods and some single cones. Since the color of oil droplets were lost in our preparation, we could not differenciate the types of single cones which were positive to iodopsin monoclonal antibodies. In some cases especially when the concentration of antibodies was relatively high, ellipsoids, cell bodies and outer limitting membrane were also stained. (2) Effects of vitamin A (VA) acid replacement on localization of iodopsin. VA acid was orally administered in VA deficient quail for about 9 weeks. The birds seemed to become blind since they did not respond to moving objects in front of them, although photoperiodic gonadal response remained positive. The retinas of these birds were stained positively with monoclonal antibodies as well as those of control birds replaced with VA acetate. (3) Effects of disruption of the retina on the localization of iodopsin. We injected $5 \mathrm{mg}$ of formoguanamine (FG), which is known to destroy the outer segments of visual cells and the pigment epithelium, to one day old quail. This treatment induced almost complete disruption of the outer segments of visual cells and the pigment epithelium when examined at 56 days of age. Behavior of the birds also indicated their blindness, although photoperiodic gonadal response and entrainment of circadian locomotor activity rhythm to light dark cycles remained almost normal. In the retinas of these birds, no positive staining by any of the monoclonal antibodies was observed, although partially disrupted cone outer segments were lightly stained.

Primary intermediates of rhodopsin, 9-cis-rhodopsin and 7-cis-rhodopsin by use of picosecond laser photolysis. Hideki KANDORI, Sinzi MATUOKA, Yoshinori SHICHIDA, Tôru YOSHIZAWA, Nobuaki NAKASHIMA ${ }^{\star}$ and Keitaro YOSHIHARA ${ }^{\star}$ Dept. of Biophysics,

Fac. of Science, Kyoto Univ. and ${ }^{\star}$ Institute of Molecular science.

The primary photochemical reaction of rhodopsin is an isomerization of 11-cis retinylidene chromophore. This isomerization proceeds rapidly and efficiently, which is closely related to the high photosensitivity of rhodopsin. In order to clarify the mechanism of the isomerization, we have studied the primary processes of rhodopsin, 9-cis- and 7-cis-rhodopsin measured by picosecond laser photolysis at room temperature.

Cattle rhodopsin $\left(\lambda_{\max }=498 \mathrm{~nm}\right)$ and 9 -cis-rhodopsin $\left(\lambda_{\max }=485 \mathrm{~nm}\right)$ were excited with a green pulse $(532 \mathrm{~nm}, 25 \mathrm{psec})$, and 7 -cis-rhodopsin $\left(\lambda_{\max }=450 \mathrm{~nm}\right)$ was excited with a blue pulse $(449 \mathrm{~nm}, 15 \mathrm{psec})$. In order to eliminate multiphoton effects due to an intense pulse, the photon density dependency was measured. In the case of rhodopsin, photorhodopsin was generated immediately after the excitation and decayed to bathorhodopsin. In the case of 9-cisrhodopsin, no clear generation of photorhodopsin was observed, while the bathointermediate displayed a similar spectrum to that of bathorhodopsin. 7-cisrhodopsin, however, was directly photo-converted to a batho-intermediate (batho-7cis-rhodopsin), which was different from bathorhodopsin. These results were in good agreement with those by low temperature spectrophotometry. Thus it was concluded as follows : Photorhodopsin is an intermediate specific to rhodopsin, which might be related only to isomerization of 11-cis-retinylidene chromophore. Bathorhodopsin is an intermediate common between rhodopsin and 9-cis-rhodopsin, then the chromophore binding site of opsin around the $\beta$-ionone ring is conserved among rhodopsin, 9-cis-rhodopsin and bathorhodopsin. The spectrum of batho-7-cisrhodopsin is located at shorter wavelength than that of bathorhodopsin. This spectral difference appears to originate from the difference in conformation of the $\beta$-ionone ring binding site between the original pigments, rhodopsin and 7cis-rhodopsin. Lumirhodopsin would be an intermediate common among rhodopsin, 9cis- and 7-cis-rhodopsins according to low temperature spectrophotometries. 
Alanine taste receptors of the puffer, Fugu pardalis. Sadao KIYOHARA*, Satoru

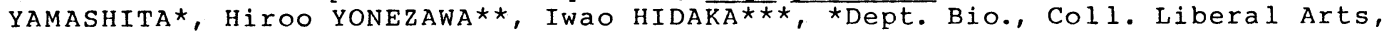
Kagoshima Univ., ** Dept. Chem., Fac. Sci., Kagoshima Univ., $\star \star \star$ Fac. Bioresources, Mie Univ.

Our previous cross-adaptation experiments show that the puffer possesses three types of different receptors for amino acids: alanine, proline and betaine receptors. The structure-activity relationships in the alanine taste receptors were examined in this study by recording the whole nerve or single fiber response to various amino acids and derivatives from the palatine nerve( ramus palatinus facialis).

Replacement of the amino or carboxyl group by other substituents such as $-\mathrm{H}$, or $-\mathrm{CH}_{3}$ always abolished the stimulating effectiveness of the molecules completely, indicating that the amino and carboxyl groups are essential for stimulating effectiveness of glycine and alanine. Esterification of the carboxyl group of the alanine or glycine significantly reduced effectiveness, but the effectiveness of methyl, ethyl or butyl ester of the two amino acids was maintained to a certain extent. This shows a negative charge on the ionized carboxyl group is not necessary. The amidation or peptideformation at the carboxyl group caused a complete or considerable loss of stimulatory effectiveness. Modification of the amino groups such as formylation or acetylation eliminated stimulatory activity. Nmethylated glycine (sarcosine, dimethylglycine) or alanine(methyl alanine) which had a positive charge on their methylated amino group were very effective for the receptors. The L-isomer was more stimulatory than its corresponding enantimer except $\mathrm{N}$-methyl alanine. An unsubstituted alpha amino group and alpha hydrogen were essential for maximal effectinevess. The side chain should be short, unbranched and uncharged for high effectiveness. These findings suggest the presence of three subunits in the receptive area. One would be involved in ionic binding to the amino group and the two others in the formation of hydrogen bridges to the carboxyl group.

The stimulating effect of 1,6-anhydro-hexopyranoses on the salt receptor of the fleshfly. I chiro SHIMADA, Hiroshi OHRUI* and Hiroshi MEGURO* Dept. Biol. Sci., Tohoku Univ. and *Dept. Food Chem., Fac. Agric., Tohoku Univ.

1,6-Anhydro-hexopyranoses were synthesized and examined their stimulating effectiveness on the labellar taste organs of the fleshfly. The salt receptor of the fly has been characterized by its specific response to cations such as salts, amino acids and organic acids.

1,6-Anhydro- $\beta-D$-galactose, 1,6 -anhydro- $\beta$-D-a1trose, 1,6-anhydro- $\beta$-D-ta1ose and 1,6-anhydro- $\beta-D$-gulose were found to stimulate the salt receptor though they are completely uncharged molecules. This was further supported by comparing the shape of evoked impulses and analizing the effects of mixed stimuli with NaC1 and other anhydro sugars on the salt and sugar receptors.

These findings may suggest the presence of a specific receptor site different from the ion channels that cause the depolarization of the salt receptor though the biological meaning of the effect of those anhydro sugars on the salt receptor is not clear. 
Medullary reticular neurons in the toad; tectal activation and axonal projection. Toshiya MATSUSHIMA, Masahiko SATOU and Kazuo UEDA. Zool.Inst., Fac. Sci., Univ. Tokyo.

Tectal efferent signals descending to the medullary/spinal regions are supposed to activate motor pattern generating mechanisms for various visuallyguided behaviors including the prey-catching behavior in amphibians. We have analyzed the neuronal pathways from the tectal efferents to the jaw- and tonguemuscle motoneurons, and it was suggested that inhibitory and excitatory interneurons in the medullary reticular formation are linking them.

In this study, to identify these interneurons, we intracellularly recorded from and stained those reticular cells which responded to the electrical stimulation of the optic tectum (OT) in paralyzed Japanese toads. Five classes of medullary neurons were morphologically identified after iontophoretic injection of cobaltic-lysine: i.e., cells in the nuc. reticularis superior (Rs), the nuc. reticularis medius (Rm), the nuc. reticularis inferior (Ri), the pretrigeminal nuc. (PTN) and the nuc. of the tractus spinalis nervi trigemini (NspV).

$\mathrm{Rm}$ axons responded to the OT stimulation with action potentials of short latencies. Spatial facilitation of these action potentials were observed when both sides of OT were simultaneously stimulated, suggesting the spatial convergence of excitatory inputs from OT onto these neurons. Somata of polygonal or bipolar shapes were located at the ventral/ventrolateral edge of the gray and had dendritic trees almost exclusively out to the white matter ventral, lateral and dorsolateral to the somata. Single axons descended through the ventral funiculus as caudally as the spinal cord. Along their course, axon collaterals emerged and projected to the gray matter or to the hypoglossal and/or spinal motor nucleus.

$\mathrm{Rs}, \mathrm{PTN}, \mathrm{Ri}$ and $\mathrm{NspV}$ cells responded to the tectal stimulation with EPSPs or mixed EPSPs-IPSPs. All of these cells had dendrites in the white matter and axons descending through the ventral/ventrolateral funiculus.

present results suggest that some of medullary reticular neurons mediate tectal descending signals to the medullary and/or spinal motor systems, thus are concerned with the command and/or motor pattern generation of the visually guided behaviors in the toad.

Influence of textured background upon motion processing in the optic tectum of Japanese toad. Masahiko SATOU, Hao-jan TSAI', Atsushi SHIRAISHI and Kazuo UEDA Zool. Inst., Fac. Sci., Tokyo Univ. and 'Dept. Biol. Sci. \& Biotech., Tsinghua Univ.

To investigate motion processing in toad's optic tectum responses of single neurons to textured and non-textured stimuli were analyzed. Consequently, following conclusions were obtained. (1) Toad's optic tectum is involved in relative motion processing. (2) Moving "object" (i.e., non-textured black or textured pattern moving in front of stationary textured background) as well as moving "hole" (i.e., textured pattern moving behind stationary textured foreground) are effective in evoking nuronal responses. (3) (i) Textured background moving behind "in-phase" with or "anti-phase" with object generally suppresses neuronal responses to the object. (ii) In-phase suppression is stronger than anti-phase suppression in most cases. (iii) Anti-phase facilitation is observed in some cases. (4) (i) Receptive field of some classes of neurons (e.g., class T5 neurons) is organized with antagonistic excitatory "center" and inhibitory "surround." (ii) Center is composed of a mixture of excitatory region and inhibitory region. (5) (i) "Motion after-response," i.e., response to stationary object due to after-effects of previously moving textured background, is found in class $\mathrm{T} 5$ as well as $\mathrm{T} 7$ neurons. (ii) This motion afterresponse is caused by neuron's rebound activity, which is derived from masking effects of the excitatory receptive field. (iii) It is supposed that the motion afterresponse is involved in sorts of perceptual motion after-effects, which are perceived by human subjects in similar experimental situations. 
The fractal dimension of the dendritic tree of cerebellar Purkinje cell during the onto- and phylogenetic developments. Toshiaki TAKEDA, Yukio KOBAYASHI and Takahide MATSUOKA Laboratory of Physiological Science, Jichi Medical School, School of Nursing and Department of Electrical Engineering, Utsunomiya University

The cerebellar Purkinje cell has a highly branched dendritic tree extending in a transverse plane to the folium. Such a two-dimensional tree pattern was subjected to fractal analysis to evaluate the intricacy of the dendritic tree. Rats and mice of ages of $7-100$ postnatal days were deeply anesthetized with nembutal and perfused transcardially with $5 \%$ glutaraldehyde and $2.4 \%$ potassium dichromate. The cerebella were processed with a protocol of rapid Golgi method. stained Purkinje cells in folium at the primary fissure, which is one of the earliest maturing region in the cerebellum, were selected and their dendritic tree patterns were traced with camera lucida. The patterns were projected onto a grid, and registered with a digitizer into computer disc files. The number of squares which traverse the patterns were counted as the grid size changed. A log-log plot of the number versus grid size showed a linear relationship indicating that the Purkinje cell dendritic tree has a statistical self-similarity. The fitness of the data to the line was evaluated by contribution ratios which were in the range of $0.985-0.999$ in the present data. The inclination of the line by least-square fitting gave the dimension (D) of the dendritic tree. D was 1.34 at 5 days old, which increased sigmoidally to 1.77 at 50 days old in the rat. Similar development was observed in the mice. The time course of increase of dimension in the rats and mice was well correlated with development of the width and area of the dendritic fields. Published dendritic patterns of Purkinje cells of the lamprey (Johnston, 1902), shark (Schaper, 1898), pearch (Schaper, 1893), turtle (Larsell, 1932), bird (Cajal, 1911) and human (Zecevic and Rakic, 1976) were also subjected to fractal analysis. Increase of dimension of Purkinje cells during evolution could be correlated to the diverging time of each species on the evolutional scale. Two regression lines, one for aquatic vertebrates and the other for terrestrial vertebrates, could be fitted to presently studied animals, which supported that these two groups took different evolutional course of cerebellar development reflecting different movement strategy.

Dead time for signal-transmission from transverse tubular membrane to sarcoplasmic reticulum in frog skeletal muscle. Yoshiaki SATO. Dept. Physiol., Natl. Def. Med. Col 1. , Tokorozawa.

The dead time (DT) in a frog (Rana japonica) single twitch fiber immobilized with stretching to a striation spacing of $3.3 \mu \mathrm{m}$ at $22^{\circ} \mathrm{C}$ was tentatively estimated from the simultaneous record of both a sarcolemmal action potential (SAP) recorded with an intracellular glass microelectrode and an arsenazo response related to the complex formation between $\mathrm{Ca}^{2+}$ released from the sarcoplasmic reticulum (SR) and an intracellularly preinjected $\mathrm{Ca}^{2+}$ indicator dye arsenazo III (Fig. 1). First, using mathematical first-order delay model, a delay constant $(\tau)$ associated with an action potential spreading along the transverse tubular ( $T$ ) system membrane ( $T$ membrane action potential, TAP) was measured from another simultaneous record of both SAP and an optical spike related to an extracellularly applied potential probe dye NK2935, which is thought to reflect TAP. One fiber immobilized with EGTA-preinjection showed $\tau=0.24 \mathrm{~ms}$ at $24{ }^{\circ} \mathrm{C}$. Based on this value, a delayed action potential simulated to TAP (Fig. 1b) was computed from the observed SAP (Fig. 1a). Secondly, the $\mathrm{Ca}^{2+}$ flux (Fig. 1C) associated with the $\mathrm{Ca}^{2+}$ release at the SR membrane was estimated by Baylor's kinetic model based on a $\mathrm{Ca}^{2+}$ transient, and as a novel approach, the $\mathrm{Ca}^{2+}$ transient (Fig. 1d) was also kinetically calculated from the observed arsenazo response (Fig. 1e). The DT was here defined as a temporal lag between the $r$ ising phase of the delayed action potential and that of the $\mathrm{Ca}^{2+} f l u x$ thus obtained and estimated $1.0 \mathrm{~ms}$ in this example, suggesting the possibility that DT may mainly be expended for physical events such as conformation changes of the dihydropyridine-receptive $\mathrm{Ca}^{2+}$ channel considered as a voltage sensor and $/$ or of a feet-substance responsible for the $\mathrm{s}$ ignal-transmission from $\mathrm{T}$ membrane to $\mathrm{SR}$.

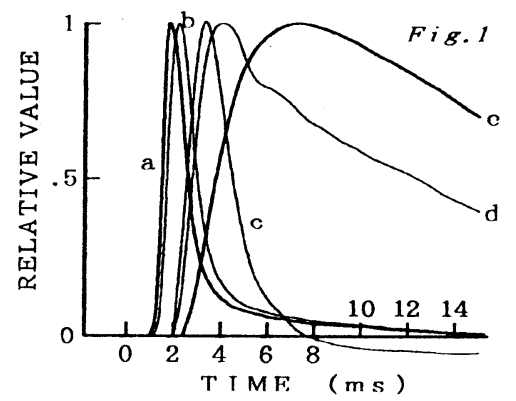


Classification of $\beta$-hydroxyglutamic acid receptors in giant neurones of an African giant snail (Achatina fulica Ferussac). Hiroshi TAKEUCHI, Kazuko WATANABE and Philipp NOVALES-LI, Dept. Physiol., Sch. Med., Gifu Univ.

Al though L-glutamic acid is not effective, $\beta$-hydroxy-L-glutamic acid ( $L-B H G A$ ) is markedly effective on several giant neurones of an African giant snai] (Achatina fulica Férussac). To elucidate the L-BHGA conformation-activity relationships and the functional roles of $\beta$-hydroxyl group, we examined effects of stereoisomers of the novel L-BHGA analogue conformationally fixed in extended or folded forms, $\alpha$-(carboxycyclopropyl)glycine (L-ACCG), on the following five neurones sensitive to L-BHGA: PON (periodically oscillating neurone), VIN (visceral intermittently firing neurone), d-RPLN (dorsal-right parietal large neurone), RAPN (right anterior pallial neurone) and V-RCDN (ventral-right cerebral distinct neurone).

The L-BHGA receptors were classified in neurone level into BHG 1,2 and 3 types, and according to the L-BHGA form acting on receptors into $\mathrm{Ex}$ (extended), Fd (folded) and Un (undetermined) types. In taking together the two classifications, these were actually BHG $1 \mathrm{Ex}, 1 \mathrm{Fd}, 2 \mathrm{Un}, 2 \mathrm{Fd}$ and $3 \mathrm{Un}$ types. The BHG 1 type receptors, existing in PON and VIN, are inhibited by L-BHGA. Of these, the $1 \mathrm{Ex}$ type was activated by erythro-L- compound in extended form, whereas the $1 \mathrm{Fd}$ type was sensitive to threo-L- compound in folded form. The role of $\beta$-hydroxyl group in L-BHGA molecule to show the effects on BHG 1 type receptors is to $\mathrm{fix}$ L-BHGA in extended (for 1Ex type) or folded (for $1 \mathrm{Fd}$ type) forms.

The BHG 2 type receptors, which exist in d-RPLN and RAPN, were excited by LBHGA. The 2 Un type was affected by erythro-L- compound, active form of which is yet undetermined, and the hydroxyl group in L-BHGA molecule acts probably as a proton donor-acceptor. On the other hand, the $2 \mathrm{Fd}$ type was activated by threo-Lcompound in folded form, and the role of $\beta$-hydroxyl group is to fix L-BHGA in folded form.

The BHG 3Un type receptors, existing in $\mathrm{V}-\mathrm{RCDN}$, were inhibited by L-BHGA. These were activated mainly by threo-L- compound, but the active form of the compound is yet undetermined.

The regulation of motility of iridophores in the brackish water floating goby, Chaenogobius annularis. Tetsuro IGA and Taichi ASARI Dept. Biol., Fac. Sci., Shimane Univ.

Light reflecting chromatophores in the skin of the brackish water floating goby, Chaenogobius annularis are of an iridophore type, which contain a number of platelets as a reflecting organelle. We found that iridophores of this fish species are motile. In response to some stimuli, these platelets move back and forth within the cells. Using isolated scale preparations of this fish species, the mechanism regulating the movements of the motile iridophores was studied. High $\mathrm{K}^{+}$solution brought dispersion of the platelets within the cells. Iridophores in scales excised from chemically sympathectomized fish became unresponsive to $\mathrm{K}^{+}$. This suggested that $\mathrm{K}^{+}$acts on presynaptic elements of the nerves controlling the cells to liberate the transmitter. Noradrenaline, which was assumed to be the transmitter, induced dispersion of the platelets. The action was not blocked by propranolol, a beta adrenolytic agent, while it was effectively inhibited by phenoxybenzamine, an alpha antagonist. This indicated that noradrenaline acts on the alpha adrenoceptors of the iridophore membrane to induce platelet dispersion. Isoproterenol caused platelet aggregation and its action was antagonized by propranolol, but not by phenoxybenzamine, suggesting that Chaenogobius iridophores possess beta adrenoceptors which function in platelet aggregation within the cells. Melatonin enhanced aggregation of the platelets in the iridophores. The action of melatonin to motile iridophores was the reverse in Odontobutis obscura and the present fish species. That is, in the former melatonin effectively induced platelet dispersion. Alpha MSH had no effect on the motile response of Chaenogobius iridophores. Forskolin, an adenylate cyclase activator, was effective in inducing aggregation of the platelets. It was concluded that the motile iridophores are under the control of the sympathetic adrenergic system and hormones, and that an increase in intracellular cyclic AMP induces platelet aggregation within the cells. 
The role of retinal-binding protein during dark regeneration of rhodopsin and retinochrome in the intact retina. Akihisa TERAKITA, Reiko HARA and Tomiyuki HARA

Dept. Biol., Fac. Sci., Osaka Univ.

The squid visual cells possess two kinds of photopigment systems, rhodopsin and retinochrome. It has been suggested that the 11-cis retinal chromophore of metaretinochrome is essential for synthesis and regeneration of rhodopsin. However, those systems are situated far away from each other within the cell, the rhodopsin found in the outer segments and the retinochrome mainly in the inner segments. In order to resolve these difficulties, we recently isolated a retinal-binding protein (RALBP) from the squid retina, and showed that the mutual exchange of retinal takes place between RALBP and meta-pigments to yield original photopigments. This suggested that RALBP may transport retinals in the visual cells, and that metarhodopsin and metaretinochrome may eventually interchange their own chromophores through the mediation of RALBP to regenerate rhodopsin and retinochrome. The present stuay was aimed at comfirming this possibility in the squid intact retina.

Enucleated eyes and their eyecups were irradiated for $2-3 \mathrm{~min}$ with orange light which is not absorbed by RALBP, and incubated in the dark. At different incubation times, the retina was fractionated into three retinal proteins, rhodopsin, retinochrome and RALBP, and assayed for retinoid isomers by HPLC. On irradiation with the eyecup, $\sim 30 \%$ of rhodopsin and $\sim 55 \%$ of retinochrome changed to their own metapigments. With the eyeball, however, 2608 of retinochrome changed to metaretinochome, showing the absence of metarhodopsin. In the ligands of RALBP in the outer segments, all-trans-retinal increased and 11-cis-retinal decreased just after irradiation of eyecup and eyeball, suggesting that RALBP might serve in the regeneration of rhodopsin from metarhodopsin produced by irradiation. In the irradiated eyecup, about half of the metarhodopsin changed back to rhodopsin during dark incubation for $1 \mathrm{hr}$. As the rhodopsin regeneration advances, metaretinochrome returned to retinochrome, showing the decrease of 11-cis and increase of all-trans in the RALBP. On the other hand, during dark incubation of irradiated eyeball, some metaretinochrome also returned to retinochrome, showing the increase of 11-cis and decrease of alltrans in the RALBP. These variations due to the amount of metarhodopsin revealed that RALBP actually acts as a shuttle for retinal in the living visual cells.

Extraction and Purification of Chicken Iodopsin with CHAPS Toshiyuki OKANO, Yoshitaka FUKADA, Igor D.ARTAMONOV and Tôru YOSHIzAWA Dept. of Biophysics, Fac. of Science, Kyoto Univ.

Chicken iodopsin is a red-sensitive color visual pigment which is only a pigment well characterized spectrophotometrically among several cone visual pigments. However, we have a little information about the visual transduction mechanism in cone cells. Recent findings suggest similarities in the transduction process between rod and cone cells, in which the first process might be an activation of transducin by a photo-bleaching intermediate of a cone visual pigment. Our first goal in this investigation is to confirm this process.

For extraction and purification of chicken cone pigments, digitonin has been used, but it tends to form a large micelle. Furthermore, it is difficult to remove digitonin from purified pigment solution due to the low critical micelle concentration value. For the purpose of the biochemical characterization of the pigment, detergents should be carefully substituted with a lipid after purification to exclude the artificial effect on the protein-protein interaction. Since cone pigments are more labile than rhodopsin, several detergents and conditions were screened for extracting cone pigments in the stable form. Then we succeeded in solubilizing rhodopsin and cone pigments from outer segment suspension of chicken retina by use of CHAPS in the presence of phosphatidylcholine. The solubilized pigments were purified by successive chromatographies on Con A- and DEAE-Sepharose columns. Thus we obtained fractions of rhodopsin (96\% purity), iodopsin (898), green pigment $(748)$, and a mixture of blue and violet pigments contaminated with iodopsin. Maximum of the difference absorption spectrum between each pigment and its retinal-oxime was roughly estimated as follows; rhodopsin: $503 \mathrm{~nm}$, iodopsin: $571 \mathrm{~nm}$, green: $505 \mathrm{~nm}$, blue: $455 \mathrm{~nm}$, violet: $425 \mathrm{~nm}$. The maximum of iodopsin in CHAPS solution $(571 \mathrm{~nm})$, rather than that in digitonin solution $(562 \mathrm{~nm})$, is very close to that in membranes $(572 \mathrm{~nm})$ obtained by means of "partial bleaching". This suggests that the conformation of iodopsin in the membranes is conserved even after solubilization with CHAPS better than that with digitonin. This is the first report of purification of cone pigments using a detergent other than digitonin. 
Synaptic transmission between the extraocular photoreceptor and its post-synaptic cells in the Onchidium ganglia. Takako NISHI, Tsukasa GOTOW and Saburo HASHIMURA Dept. Physiol., Fac. Med., Kagoshima Univ.

It is known that an extraocular photoreceptor, the photoresponsive neurone (A-P-1) which is depolarized most effectively with $490 \mathrm{~nm}$ light, lies on the abdominal ganglion of the marine gastropod mollusc, Onchidium verruculatum. We also found that the light-induced depolarization was produced in another identified neurone (Es-1) in the same ganglion, having an action spectrum peak at $580 \mathrm{~nm} 1$ ight. This depolarizing, photoresponse of Es-1 was still maintained with exposure to the high $\mathrm{Mg}$, low $\mathrm{Ca}$, suggesting the photoresponse endogenous to the Es-1.

When the intracellular recordings of membrane potential were made simultaneously from $A-P-1$ and Es-1, Es-1 showed inhibitory post-synaptic potentials following spikes in A-P-1 elicited by intrasomatic stimulation. However, no excitatory or inhbitory post-synaptic potentials were observed in A-P-1 when Es-1 was fired. These suggested that the Es-1 received an inhibitory synaptic input from the A-P-1, but the reverse was not true.

On the other hand, the inhibitory synaptic potentials, superimposed on the depolarizing (generator) potentials were produced in Es-1 during white light stimulation to the whole ganglion, but $490 \mathrm{~nm}$ light evoked only the inhibitory synaptic potentials. The A-P-1 was depolarized by the white light, but the 1 ight of $580 \mathrm{~nm}$ had no effect on the membrane potential. However, the inhibitory potentials of Es-1 to the white and $490 \mathrm{~nm}$ lights were blocked by the high $\mathrm{Mg}$, low $\mathrm{Ca}$ saline or by adding atropine (1 $\mathrm{mM})$. Furthermore, Es-1 was specifically hyperpolarized (inhibited) by $\mathrm{ACh}$, and atropine antagonized the specific effect of ACh.

These results suggested that Es-1 responding directly to light with depolarization also received the inhibitory (hyperpolarizing) synaptic input mediated by ACh from A-P-1. Thus, it will be expected that the excitatory effect by light of Es-1 cancels out the inhibitory one by addition of ACh, and so was the fact.

Finally, it seems that the output of two extraocular photoreceptors composed of $\mathrm{A}-\mathrm{P}-1$ and Es-1 signals the difference of wavelengths in natural sunlight rather than its brightness.

Inhibitory peptides isolated from Mytilus pedal ganglia. Tatsumi HIRATA, Ichiro KUBOTA*, Norio IWASAWA**, Ikuo TAKABATAKE $\star \star \star$, Tetsuya IKEDA and Yoji ro MUNEOKA Fac. Integrated Arts and Sciences, Hiroshima Univ., *Suntory Inst. Biomed. Res., $\star \star$ Suntory Inst. Fundment. Res., and $* \star \star$ Dept. Biol., Fac. Sci., Shimane Univ.

Hirata et al. (1986) obtained two species with biological activity from the gel-filtrated acetone extracts of the pedal ganglia of Mytilus edulis. One showed relaxing action on catch tension of the anterior byssus retractor muscle of the mussel, and the other showed inhibitory action on phasic contraction of the muscle in response to repetitive electrical pulses of stimulation. Further, they suggested that these bioactive substances are peptides. Recently, the former bioactive substance which was termed catch-relaxing peptide was determined to be a heptapeptide having the following primary structure: H-Ala-Met-Pro-Met-Leu-ArgLeu-NH2 (Hirata et al., 1987).

In the present study, we purified the latter bioactive substance and indicated that it consists of two congeneric hexapeptides. We termed these peptides Mytilus inhibitory peptides (MIPs). The primary structures of MIPs are as follows:

$$
\begin{aligned}
& \mathrm{H}-\mathrm{Gly} \text {-Ser-Pro-Met-Phe-Val-NH } 2 \text { ' (Ser }{ }^{2}-\mathrm{MIP} \text { ) } \\
& \mathrm{H}-\mathrm{Gly} \text {-Ala-Pro-Met-Phe-Val-NH2 (Ala2-MIP) }
\end{aligned}
$$

These hexapeptides show inhibitory action on various molluscan muscles, such as the cardiac muscle of Meretrix lusoria and the penis retractor muscle of Achatina fulica, suggesting that they and their analogues may be widely distributed in Mollusca.

Hirata, T. Kawahara, A. and Muneoka, Y. (1986) Hiroshima J. Med. Sci. 35, $397-402$.

Hirata, T., Kubota, I., Takabatake, I., Kawahara, A., Shimamoto, N. and Muneoka, Y. (1987) Brain Res. 422, 374-376. 
Mechanisms of the control of heart beat in a mollusc, Achatina fulica Férussac. -Effects of serotonin and neuropeptides- Katsuhiko HORI and Makoto KOBAYASH Physiol. Lab., Fac. Integrated Arts and Sci.,

Hiroshima Univ.

In the central nervous system of the African giant snail, Achatina fulica Férussac, several heart regulatory neurons have been identified and their interconnections have been described. In the present experiments, the mechanisms of the heart regulation by the neurons named "periodically oscillating neuron" (PON) and "tonically autoactive neuron" (IAN) were studied by using a preparation consisted of ganglia-intestinal nerve-atrium.

Intracellular recording and stimulation of neurons were carried out by a single microelectrode, and the heart beat was recorded by a strain gauge.

The excitatory response of heart beat to firings of PON was depressed by methysergide which also blocked the potentiation of heart beat produced by serotonin. This may suggest a transmitter from PON to the heart to be serotonin. In contrast, the potentiation of heart beat by the increase of firing frequency of TAN was not af $f$ ected by methysergide. Effects of several FMRFamide-related peptides and the other neuropeptides on the heart beat were tested. FMRFamide enhanced the heart excitatory responses to both PON stimulation and serotonin application. Small cardioactive peptide $\mathrm{B}\left(\mathrm{SCP}_{B}\right)$, on the contrary, depressed the both responses. The potentiation of heart beat by IAN stimulation was not affected by the neuropeptides examined. Catch-relaxing peptide (CARP) and Mytilus inhibitory peptides (MIPs) isolated from Mytilus ganglia had no effects on the heart beat.

These results suggest that FMRFamide and $\mathrm{SCP}_{\mathrm{B}}$ are modulating excitatorily and inhibitorily, respectively, the action of main transmitter serotonin at the synapse from PON to the heart.

Impulse activity of ligamental nerves distributed in pericardial cavity of the lobster, Panulirus japonicus. Taketeru KURAMOTO Inst. Biol. Sci., Tsukuba Univ.

Ligamental nerves of Panulirus originate the 3 rd roots of thoracic ganglia and terminate near ostia as extensions of the pericardial neurohemal system. I examined what stimuli were related to firing of the ligamental nerve.

Dorsal pericardium of the lobster was removed after blood was replaced by Pantin's saline. The pericardial cavity was perfused with the saline during the experiments at $20 \pm 1^{\circ} \mathrm{C}$. Electrical activities of the anterior, medial and posterior ligamental nerves (ALN, MLN, PLN) and the heart in situ were recorded with glass suction electrodes. To stimulate the neurohemal system, the cooled saline $\left(5^{\circ}-15^{\circ} \mathrm{C}\right)$ was perfused through the pericardial cavity for 2 or 3 minutes. The saline containing serotonin or octopamine $(1 \mathrm{mM}, 1 \mathrm{ml})$ and the serum of the lobster (1 ml) were also poured into the pericardial cavity to see neurohormonal effects. A light stimulus (200 lux) was applied to the whole body by switching on the room light.

In response to a cool stimulus $\left(\mathrm{e} . \mathrm{g} .15^{\circ} \mathrm{C}\right)$, a train of impulses was produced in each of the ALN, MLN and PLN, while the heart rate decreased (e.g. from 55 to 35 beat/min). Three axons in the ligamental nerve usually fired synchronously. Burst discharges composed of several kinds of impules were sometimes produced in the ligament nerve by much cooler stimuli $\left(5\right.$ or $\left.10^{\circ} \mathrm{C}\right)$. Nerve impulses started by cooling could be recorded from the 3 rd roots in the preparation removing the head, abdomen and all viscera. These may suggest that cool receptors are in the thoracic ganglia and that pericardial neurohormones release as body temperature is decreased. After the light stimulus was applied, firing rate of the spontaneously active ALN increased for one or two minutes and returned to the initial level at three minutes. Therefore, releasing rate of pericardial hormones may increase transiently if light intensity in environment of the lobster increases suddenly.

Several kinds of impulses were initiated in ALN after the application of serotonin, octopamine or serum of the lobster. Large impulses ceased in a short period (i.e. $10 \mathrm{sec}$ ), while small impulses continued for a long period (1 minute or more). It has been suggested that the ligamental nerve releases serotonin and octopamine. Therefore, the neurosecretory cell fired by serotonin or octopamine may be stimulated by itself. 
Electrical activity of the cerebral neurosecretory cells during the head critical period in 4 th instar larvae of the tobacco hornworm, Manduca sexta. Kenji TOMIOKA and Walter E. BOLLENBACHER* Biol. Inst., Yamaguchi Univ. and Dept. Biol., Univ. North Carolina

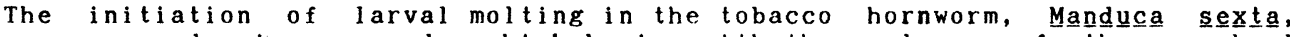
involves an endocrine cascade which begins with the release of the cerebral prothoracicotropic hormone (PTTH) from the retrocerebral corpora allata (CA). During the 4 th larval instar when the gated release of PTTH occurs, the in situ electrical activity of the nervi corporis cardiacum (NCC I+II), the paired nerve trunks for brain ( $\mathrm{Br}$ ) innervation of the corpora cardiaca (CC) and CA, undergoes a distinct change in firing pattern. The initial change in pattern, from a steady firing, was a brief intense burst of relatively large units. A short period of completely suppressed activity $(3-10 \mathrm{~min})$ followed, after which activity resumed in the form of bursts of several kinds of units previously not active. Depending upon the preparation, this event was either long-lasting (hours) or short (minutes) in duration. Preliminary examination of PTTH activity released from the CA, with in vitro assay for the hormone, showed that PTTH activity was detectable only after the long-lasting bursts, suggesting this bursting pattern was a neurophysiological event associated with. PTTH release. This bursting pattern occurred at a particular time of day during the night, and was expressed by the $\mathrm{Br}-\mathrm{CC}-\mathrm{CA}$ both in si $\underline{\mathbf{t}} \underline{\mathrm{u}}$ and in vitro. The fact implies that the characteristic bursting pattern is driven by a clock mechanism which resides in the $\mathrm{Br}-\mathrm{CC}-\mathrm{CA}$ and works even in vitre. The complexity of the bursting pattern around the time of PTTH release suggests that a variety of cell types may be involved in a coordinated, transient inhibition of electrical activity of the NCC I+II that is followed by a selective disinhibition of a specific set of cerebral neurons culminating in PTTH release.

Light and food as environmental cues to entrain the locomotor activity rhythm of a loach, Misgurnus anguillicaudatus. Mayum1 NARUSE and Tadashi OISHI, Dept. Biol., Fac. Sci., Nara Women's Univ.

Light (light-dark cycle) is a well known zeitgeber for the entrainment of circadian locomotor activity rhythm of various animal species. However, investigations on the other environmental cues and the interactions among these zeitgebers are meagre. In the present study, we investigated the effect of scheduled feeding on the locomotor activity rhythm of a loach, Misgurnus anguillicaudatus, and the interaction between light-dark cycles and scheduled feeding. Male and female loaches (10-17cm in body length) were held singly in a water tank placed in a bioclimatic room $\left(25 \pm 1^{\circ} \mathrm{C}\right)$. Locomotor activity rhythms were recorded with an event-recorder and a digital data recorder through an infrared phototransister system. (1) Effects of light and food given in phase or in opposite phase on the locomotor activity pattern. When loaches were fed at scheduled feeding time at $12: 00$ in the dark phase of LD cycle. (LD $12: 12$; light on from 18:00 to $6: 00$ ), all of the six fish exhibited greater locomotor activity in dark (nocturnal activity). However, when the food was given at 12:00 in the light phase of LD cycle (LD 12:12; light on from 6:00 to 18:00), two activity peaks (one in light before meal time (anticipatory activity) and another after light off) appeared in most of the fish. In some fish, the activity in dark disappeared gradually and only the anticipatory activity in light before meal time remained, indicating the change of activity pattern from nocturnal to diurnal. (2) Effects of one zeitgeber on the locomotor activity pattern. When the fish were raised under LD 12:12 without food, three out of six fish were active in dark period, one fish was active in light period and the other two reduced their activity significantly in 1-3 days after starvation. Since variable results were obtained depending on the previous conditions, there seems to be after-effect of the previous condition. When feeding was scheduled in constant darkness, all the fish were entrained to feeding time with typical resting time after meal. Thus, both LD cycle and scheduled feeding affect locomotor activity pattern of the loach and seem to be effective as zeitgebers to entrain the locomotor activity rhythm. 
Spontaneous disintegration of hydra into single cells induced by hypertonic conditions. Takao SHIOYA and Hiromasa KIJIMA Dept. Biol., Fac. Sci., Kyushu Univ.

We found that the whole animal of hydra disintegrates spontaniously and gives viable singles cells, when exposed to appropriate hypertonic solutions. For example, when the whole animal of Hydra japonica was put into a salt solution

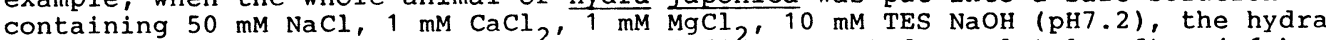
began to disintegrete whithin an hour and disintegrated completely after 4-6 hr treatment. During the disintegration process, the cells were detached from the animal and fell down to the bottom of the vessel, retaining the intact and native shapes of every types of cells for a few hours. That is, the epitheriomuscular cell had long processes of myoneme, the nematoblasts were in nests of 8-32 cells, nematocytes had cnidocils and battery cells in crescent-shape contained regularly arranged nematocytes. The viability of these cell are acertained by tripan-blue staining. These intact shapes of single cells have been observed so far only on the dead cells obtained by maceration technique (David, 1973).

The disintegration occurred in almost all-or-none fashion, dependent on the concentration $\mathrm{NaCl}$ in the above solution. Below $40 \mathrm{mM} \mathrm{NaCl}$ (the threshold) hydra kept its intact shape for a few days, while above $40 \mathrm{~mm}$, disintegrated completely within a day. The speed and the threshold concentration varied, dependent on the kinds of anions and cations. In the solution containing acetate instead of $\mathrm{Cl}^{\text {, }}$ the threshold was much higher and the disintegration proceeded much slower. A quantitative evaluation of the disintegration process by counting the detached cells showed that the effects of $\mathrm{Cl}^{-}$and acetate were not additive. Acetate ion depressed the effect of $\mathrm{Cl}^{-}$. Sucrose added instead of $\mathrm{NaCl}$ could also induce distintegration.

CDNA cloning and sequencing of squid retinal-binding protein. Koichi OZAKI, Akihisa TERAKITA, Reiko HARA, Tomiyuki Hara, Mikio NISHIMURA*, Ikuko HARA-

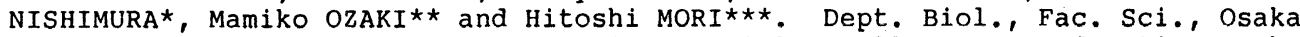
Univ., *Dept. Biol., Fac. Sci. and $\star \star$ Dept. Biol., College Gen. Education, Kobe Univ. and $\star \star \star$ Meiji Inst. Health Sci.

In the cephalopod eye, the photoreceptor cells have a dual system of retinalbearing photopigments, rhodopsin and retinochrome. When retinochrome absorbs light, its retinaldehyde chromophore is isomerized from the all-trans to 11-cis form. As this isomerization is the reverse of what occurs upon irradiation of rhodopsin, we suggested that the photoproduct of retinochrome may act as a supplier of 11 -cis retinal required for rhodopsin formation. However, we had not yet known whether those pigments in the membrane directly contact with each other or a mediator carries retinal between them.

A few years ago, we found a novel retinaldehyde-binding protein (RALBP) in the soluble fraction of the retinal homogenate of the squid, Todarodes pacificus. Recent studies on RALBP have shown that the protein is located in both rhabdomal layer and nucleated cell bodies, and that chromophore exchange occurs between metarhodopsin and RALBP, and between RALBP and metaretinochrome. As these findings suggest that RALBP play an important roles on rhodopsin formation in the visual cells, we began to study the molecular architecture of RALBP.

The analysis of in vitro translation directed by squid retinal RNA revealed that the molecular weight of in vitro synthesized RALBP is the same as that of mature protein, proving that RALBP is not derived from a larger precursor.

Expression cDNA library of the squid retina was screened with rabbit antiRALBP IgG. Five immunochemically positive clones were isolated from 10 recombinants, and 3 of them produced the immunoreactive polypeptide with a little larger molecular weight than the mature RALBP. This suggests that the cDNA inserts of those clones include the complete coding region of the mRNA.

The nucleotide sequence of the CDNA was then determined. mRNA of RALBP contains an extensive non-coding region at the $3^{\prime}$ end. The primary structure of the protein deduced from the nucleotide sequence so far reveals no homology to any other proteins, suggesting that squid RALBP belongs to a new family of protein. 
Attempt to isolate the cDNA clone of chicken iodopsin by use of monoclonal antibodies. Osamu KUWATA, Yoshitaka FUKADA, Yoshinori SHICHIDA, TOrU YOSHIZAWA, Kunio YASUDA and Yoshiro SHIMURA. Dept. of Biophys, Fac. of Sci, Kyoto Univ., Kyoto 606 , JAPAN.

Chicken iodopsin is a red-sensitive color visual pigment, whose absorption maximum ( $562 \mathrm{~nm}$; in the presence of chloride ion) is located at longer wavelength than that of chicken rhodopsin $(501 \mathrm{~nm})$. Though some characteristics of iodopsin have been investigated by biochemical and spectroscopic techniques, the primary structure has not yet been determined. Judging from the deduced amino acid sequences of human visual pigments, the structure of iodopsin should be considerably different from that of rhodopsin. As the first step of investigating the structure of iodopsin and its physiological function in the cone visual cell, we have prepared four kinds of monoclonal antibodies, using the purified iodopsin $(34 \mathrm{kDa})$ as an antigen for both immunization of mice and screening of antibody-secreting hybridomas. They proved to be so specific for iodopsin as to enable us to isolate an iodopsin CDNA clone from a cDNA library of chick retina in which iodopsin has been confirmed to exist. Then we can deduce the amino acid sequence of iodopsin from the nucleotide sequence of the cDNA. From total RNAs of freshly excised chick retinas, poly(A) ${ }^{+}$RNAs were separated by an oligo(dT) affinity column. Double-stranded cDNA(complementary DNA) s of the poly(A)+ RNAs were synthesized and cloned into the expression vector, gt11, followed by in vitro packaging for the construction of a cDNA library of chick retina.

Plaques bearing the expressed product of each cDNA were transferred to nitrocellulose membranes, and then screened using a mixture of iodopsin-specific antibodies as a probe. Six cDNA clones showed positive signals. A northern blot analysis revealed that one of the cDNA clones of $0.6 \mathrm{~kb}$ specifically hybridized with a mRNA (about $2.2 \mathrm{~kb}$ ) in the chick retina but not with those in liver and kidney. These observations suggest that this clone contains the coding region of iodopsin gene. 